Draft version August 13, 2013

Preprint typeset using $\mathrm{IAT}_{\mathrm{E}} \mathrm{X}$ style emulateapj v. 5/2/11

\title{
DWARF GALAXIES WITH OPTICAL SIGNATURES OF ACTIVE MASSIVE BLACK HOLES
}

\author{
Amy E. REINES ${ }^{1}$ \\ National Radio Astronomy Observatory, Charlottesville, VA 22903, USA \\ Jenny E. GREene \\ Department of Astrophysical Sciences, Princeton University, Princeton, NJ 08544, USA \\ AND \\ MARLA GeHA \\ Department of Astronomy, Yale University, New Haven, CT 06520, USA \\ Draft version August 13, 2013
}

\begin{abstract}
We present a sample of 151 dwarf galaxies $\left(10^{8.5} \lesssim M_{\star} \lesssim 10^{9.5} \mathrm{M}_{\odot}\right)$ that exhibit optical spectroscopic signatures of accreting massive black holes (BHs), increasing the number of known active galaxies in this stellar mass range by more than an order of magnitude. Utilizing data from the Sloan Digital Sky Survey Data Release 8 and stellar masses from the NASA-Sloan Atlas, we have systematically searched for active BHs in $\sim 25,000$ emission-line galaxies with stellar masses comparable to the Magellanic Clouds and redshifts $z<0.055$. Using the narrow-line $[\mathrm{O}$ III $] / \mathrm{H} \beta$ versus $[\mathrm{N} \mathrm{II}] / \mathrm{H} \alpha$ diagnostic diagram, we find photoionization signatures of BH accretion in 136 galaxies, a small fraction of which also exhibit broad $\mathrm{H} \alpha$ emission. For these broad-line AGN candidates, we estimate $\mathrm{BH}$ masses using standard virial techniques and find a range of $10^{5} \lesssim M_{\mathrm{BH}} \lesssim 10^{6} \mathrm{M}_{\odot}$ and a median of $M_{\mathrm{BH}} \sim 2 \times 10^{5} \mathrm{M}_{\odot}$. We also detect broad $\mathrm{H} \alpha$ in 15 galaxies that have narrow-line ratios consistent with star-forming galaxies. Follow-up observations are required to determine if these are true type 1 AGN or if the broad $\mathrm{H} \alpha$ is from stellar processes. The median absolute magnitude of the host galaxies in our active sample is $M_{g}=-18.1 \mathrm{mag}$, which is $\sim 1-2$ magnitudes fainter than previous samples of AGN hosts with low-mass BHs. This work constrains the smallest galaxies that can form a massive $\mathrm{BH}$, with implications for $\mathrm{BH}$ feedback in low-mass galaxies and the origin of the first supermassive BH seeds.
\end{abstract}

Subject headings: galaxies: active — galaxies: dwarf — galaxies: nuclei — galaxies: Seyfert

\section{INTRODUCTION}

Over the past decade we have come to appreciate that nuclear black holes $(\mathrm{BHs})$ with $M_{\mathrm{BH}} \sim 10^{6}-10^{9} \mathrm{M}_{\odot}$ are a ubiquitous component of massive galaxies in the modern Universe (e.g., Kormendy \& Richstone 1995: Kormendy \& Ho 2013), yet the origin of the first highredshift supermassive BH "seeds" is far from understood. Observations of quasars with billion solar-mass BHs at a time when the Universe was less than a Gyr old (e.g., Fan et al. 2001; Mortlock et al. 2011) demonstrate that supermassive BHs almost certainly started out with masses considerably in excess of normal stellar-mass BHs. However, we do not know how these initial seed $\mathrm{BHs}$ formed in the early Universe, how massive they were originally, or in what types of galaxies they formed. While direct observations of distant seed BHs and their host galaxies in the infant Universe are unobtainable with current capabilities, nearby dwarf galaxies are within observational reach and can provide important constraints on the formation path, masses and hosts of $\mathrm{BH}$ seeds (e.g., Volonteri 2010 Greene 2012). The goal of this work is to systematically search for optical AGN signatures in dwarf galaxies $\left(M_{\star} \lesssim 3 \times 10^{9} \mathrm{M}_{\odot}\right)$, where very few mas-

areines@nrao.edu

${ }^{1}$ Einstein Fellow sive $\mathrm{BH} \mathrm{2}^{2}$ have hitherto been found.

The growth of supermassive BHs appears to be linked to the evolution of their hosts, with more massive galaxies generally harboring more massive $\mathrm{BHs}$ (e.g., Gebhardt et al. 2000a Ferrarese \& Merritt 2000, Marconi \& Hunt 2003 Gültekin et al. 2009, McConnell \& Ma 2013). Therefore, unlike today's massive galaxies, lowmass dwarf galaxies with relatively quiet merger histories may host $\mathrm{BHs}$ with masses not so different from the first seed BHs (Bellovary et al. 2011). Models of BH growth in a cosmological context (Volonteri et al. 2008) indicate that if we can determine the $\mathrm{BH}$ occupation fraction and mass distribution in present-day dwarf galaxies, we will gain insight into whether seed BHs formed primarily as the end-product of Population III stars (e.g., Bromm \& Yoshida 2011), in a direct collapse scenario (e.g., Haehnelt \& Rees 1993; Lodato \& Natarajan 2006 Begelman et al. 2006), or in a runaway accretion event at the center of a dense star cluster (e.g., Portegies Zwart et al. 2004, Miller \& Davies 2012). Studying the scaling relations between $\mathrm{BHs}$ and galaxies at low-mass may provide further clues (e.g., Volonteri \& Natarajan 2009).

There are additional reasons to search for and study

${ }^{2}$ We use the term "massive $\mathrm{BH}$ " to refer to BHs larger than normal stellar-mass BHs. BHs with masses in the range $\sim 10^{4}-$ $10^{6} \mathrm{M}_{\odot}$ are also sometimes referred to as "low-mass BHs" (meaning relatively low-mass supermassive $\mathrm{BHs}$ ) or "intermediate-mass BHs (IMBHs)." 
massive BHs in dwarf galaxies. Understanding the radiative properties of $\mathrm{BHs}$ in this mass range is important for models of the impact of mini-quasars on reionization and the formation of the first galaxies (e.g., Milosavljević et al. 2009). Moreover, the study of BH accretion and star formation in low-mass dwarfs today may prove a good laboratory for understanding their interplay at early times (e.g., Reines et al. 2011; Jia et al. 2011, Alexandroff et al. 2012) and the apparent contribution of obscured AGNs in blue low-mass galaxies to the cosmic X-ray background (Xue et al. 2012). Determining the presence of AGN in low-mass galaxies will also help constrain $\mathrm{BH}$ feedback and galaxy formation models at all mass scales. For instance, while BH feedback is often invoked to explain star formation quenching in massive galaxies (e.g., Croton et al. 2006, Kimm et al. 2009), the quenched fraction of central galaxies decreases with stellar mass (Wetzel et al. 2012) and may become zero below a stellar mass near $10^{y} \mathrm{M}_{\odot}$ (Kauffmann et al. 2003a; Geha et al. 2012). Finally, signatures of tidal disruptions of stars are strongest for low-mass BHs (e.g., Strubbe \& Quataert 2009, Rosswog et al. 2009, Miller \& Gültekin 2011) and $\sim 10^{5} \mathrm{M}_{\odot} \mathrm{BH}$ mergers have the right frequency to provide a signal for future gravitational wave experiments (e.g., Hughes 2002).

The most secure method of discovering supermassive BHs uses stellar or gas dynamics to weigh the central BH (see review in Kormendy 2004). However, the gravitational sphere of influence cannot be resolved for low-mass BHs in small galaxies much beyond the Local Group. For example, $r_{\text {infl }}=G M_{\mathrm{BH}} / \sigma^{2} \sim 0.5$ pc assuming $M_{\mathrm{BH}} \sim 10^{5} \mathrm{M}_{\odot}$ and a stellar velocity dispersion of $\sigma \sim 30 \mathrm{~km} \mathrm{~s}^{-1}$. Even with the resolution afforded by the Hubble Space Telescope (HST) or adaptive optics on large ground-based telescopes, such a small radius of influence can only be resolved out to a distance of $\sim 1 \mathrm{Mpc}$. Nevertheless, there are limits on dynamical BH masses and a couple of detections in nearby low-mass galaxies. The Local Group galaxies NGC 205 (a bright early-type dwarf satellite of Andromeda), Ursa Minor, and Fornax (both classical dwarf spheroidals around the Milky Way) all have upper limits of $M_{\mathrm{BH}} \lesssim$ a few $\times 10^{4} \mathrm{M}_{\odot}$ from stellar dynamics (Valluri et al. 2005 , Lora et al. 2009, Jardel \& Gebhardt 2012). In contrast, the low-mass elliptical M32 has a BH with $M_{\mathrm{BH}} \sim$ a few $\times 10^{6} \mathrm{M}_{\odot}$ (e.g., Dressler \& Richstone 1988, van der Marel et al. 1998). NGC 404, a low-mass S0 just beyond the Local Group, also has a likely $\mathrm{BH}$ with $M_{\mathrm{BH}} \sim 5 \times 10^{5} \mathrm{M}_{\odot}$ derived from molecular hydrogen gas kinematics (Seth et al. 2010). A number of very late-type spiral galaxies have dynamical BH mass limits, including M33 with $M_{\mathrm{BH}} \lesssim$ a few $\times 10^{3} \mathrm{M}_{\odot}$ (Gebhardt et al. 2001). Outside the Local Group, Barth et al. (2009) find a conservative upper limit of $M_{\mathrm{BH}} \lesssim 3 \times 10^{6} \mathrm{M}_{\odot}$ on the active $\mathrm{BH}$ in NGC 3621, while Neumayer \& Walcher (2012) find upper limits of $\sim 10^{6} \mathrm{M}_{\odot}$ for nine more late-type spirals.

In more distant systems, and in particular those with low-mass BHs, obtaining dynamical evidence for a $\mathrm{BH}$ is not feasible and we must rely on radiative signatures of AGN powered by $\mathrm{BH}$ accretion. The dwarf spiral galaxy NGC $4395\left(M_{\star} \sim 1.3 \times 10^{9} \mathrm{M}_{\odot}\right)$, for example, has clear and unambiguous evidence for a central $\mathrm{BH}$ from highionization narrow emission lines and broad permitted lines in the optical (Filippenko \& Sargent 1989), a compact radio jet (Wrobel \& Ho 2006), and extreme variability in the X-ray (Vaughan et al. 2005; Moran et al. 2005). NGC 4395 is not just a fluke; POX 52 is dwarf elliptical $\left(M_{\star} \sim 1.2 \times 10^{9} \mathrm{M}_{\odot} ;\right.$ Thornton et al. 2008) with an AGN that is very similar in optical properties to NGC 4395 (Kunth et al. 1987; Barth et al. 2004). These two dwarf galaxies have $\overline{\mathrm{BH}}$ mass estimates of $M_{\mathrm{BH}} \sim 3 \times 10^{5} \mathrm{M}_{\odot}$ (Filippenko \& Ho 2003; Peterson et al. 2005, Thornton et al. 2008).

Reines et al. (2011) present multi-wavelength evidence for the first example of a massive $\mathrm{BH}$ in a dwarf starburst galaxy, Henize 2-10, which has an irregular central morphology and no discernible bulge. The evidence for an accreting massive BH includes Very Large Array radio and Chandra hard X-ray point sources at the center of the galaxy and clearly separated from the bright H II regions. Follow-up Very Long Baseline Interferometry (VLBI) observations reveal parsec-scale non-thermal radio continuum from the precise location of the putative active nucleus (Reines \& Deller 2012) and no star clusters are seen at this location in HST observations. The source lies at the center of a $\sim 250$-pc-long ionized gas structure that is suggestive of bipolar flow and the position of the central source is consistent with the dynamical center of the galaxy. Reines et al. (2011) estimate a BH mass of $\log \left(M_{\mathrm{BH}} / \mathrm{M}_{\odot}\right)=6.3 \pm 1.1 \mathrm{using}$ the radio-X-ray $-M_{\mathrm{BH}}$ fundamental plane (Merloni et al. 2003), and Kormendy \& Ho (2013) estimate that Henize 2-10 has a stellar mass of $M_{\star} \sim 1.4 \times 10^{9} \mathrm{M}_{\odot}$ with an uncertainty of a factor of 3. To determine the frequency of objects like NGC 4395, Pox 52, and Henize 2-10 requires large surveys for AGN signatures in low-mass galaxies.

In an effort to find AGNs with low-mass BHs, Greene \& Ho (2004, 2007a), and Dong et al. (2012) searched systematically through the Sloan Digital Sky Survey (SDSS; York et al. 2000) for broad $\mathrm{H} \alpha$, as a signature that an accreting $\mathrm{BH}$ is present. They present samples of $>200$ galaxies with $M_{\mathrm{BH}} \lesssim 10^{6.5} \mathrm{M}_{\odot}$ and median $\mathrm{BH}$ masses of $M_{\mathrm{BH}} \sim 1 \times 10^{6} \mathrm{M}_{\odot}$. In a complementary search, Barth et al. (2008) present a sample of narrow-line AGN in host galaxies with relatively low stellar velocity dispersions, suggesting the presence of low-mass BHs. However, the vast majority of galaxies in all of these samples are more massive than $M_{\star} \sim 10^{9} \mathrm{M}_{\odot}$, and thus do not probe the dwarf galaxy regime (Barth et al. 2008; Greene et al. 2008; Jiang et al. 2011). Determining the true space densities of low-mass BHs from the SDSS surveys is very difficult because of the joint bias towards luminous galaxies (for spectroscopic targeting) and luminous AGNs (for spectroscopic identification; Greene \& Ho 2007b). Searches based on mid-infrared (e.g., Spitzer spectroscopy; Satyapal et al. 2007, 2008, 2009; Goulding et al. 2010) or X-ray observations (Ghosh et al.|2008. Desroches \& Ho 2009; Gallo et al.|2008, 2010; Kamizasa et al. 2012; Miller et al. 2012, Schramm et al. 2013) have so far covered only small volumes, often with heterogeneous selection.

So far there has been no systematic look at the AGN population in dwarf galaxies. Previous AGN studies have looked at galaxies with stellar masses typically larger than $\sim 10^{10} \mathrm{M}_{\odot}$. Here we undertake the first systematic search for active massive BHs in dwarf galaxies with 
stellar masses comparable to or less than the Large Magellanic Cloud (LMC). Starting with a sample of $\sim 25,000$ emission-line galaxies in the SDSS Data Release 8 (DR8) spectroscopic catalog (Aihara et al. 2011) with stellar masses $M_{\star} \lesssim 3 \times 10^{9} \mathrm{M}_{\odot}$ and redshifts $z<0.055$, we identify dwarf galaxies with narrow-line photoionization signatures of an accreting massive $\mathrm{BH}$. In addition, we search for broad $\mathrm{H} \alpha$ emission that may indicate gas orbiting in the deep potential well of a massive BH. Collectively, the galaxies presented here are the smallest and least-massive known to contain massive BHs.

\section{DATA AND DWARF GALAXY SAMPLE}

We have selected our sample of dwarf galaxies from the NASA-Sloan Atlas ${ }^{3}$ (NSA), which in turn is based on the SDSS DR8 spectroscopic catalog (York et al. 2000 Aihara et al. 2011). We use the NSA for selecting our parent sample of galaxies and investigating galaxy properties, but analyze the SDSS spectra with our own customized software to search for signatures of $\mathrm{BH}$ accretion (Section 3). The SDSS uses the dedicated 2.5-meter wide-field Sloan Foundation Telescope and a 640-fiber double spectrograph at Apache Point Observatory in New Mexico (Gunn et al. 2006). The instrumental fiber diameter is $3^{\prime \prime}$ and the spectrophotometrically calibrated spectra cover a wavelength range from 3800 - $9200 \AA$, with a instrumental dispersion of $69 \mathrm{~km} \mathrm{~s}^{-1}$ per pixel.

The NSA provides a re-analysis of the SDSS imaging and spectroscopic data for all galaxies with redshifts $z<0.055$. Photometry is improved over the standard SDSS DR8 photometric catalog as described in Blanton et al. (2011) and results in a cleaner sample of dwarf galaxies compared to previous catalogs that include many fragmented pieces of extended massive galaxies. The spectroscopic data is re-analyzed using the methods of Yan \& Blanton (2012) and Yan (2011). We only use the emission line measurements in the NSA for quality cuts in signal-to-noise and equivalent width as described below. In addition to a variety of galaxy parameters, estimates of stellar masses are provided in the NSA. Stellar masses are derived from the kcorrect code of Blanton \& Roweis (2007), which fits broadband fluxes using templates based on the stellar population synthesis models of Bruzual \& Charlot (2003) and the nebular emission-line models of Kewley et al. (2001). Masses are given in units of $\mathrm{M}_{\odot} h^{-2}$ and we have assumed $h=0.73$.

Since our goal is to search for dwarf galaxies hosting active massive BHs, we first select sources in the NSA with stellar masses $M_{\star} \leq 3 \times 10^{9} \mathrm{M}_{\odot}$ and obtain 44,594 objects. Our mass threshold, while somewhat arbitrary, is approximately equal to the stellar mass of the LMC (van der Marel et al. 2002), which is the most massive dwarf galaxy satellite around the Milky Way. While we do not apply a minimum stellar mass limit, nearly all of the galaxies in our parent sample have $M_{\star} \gtrsim 10^{7} \mathrm{M}_{\odot}$ due to the SDSS spectroscopic apparent magnitude limit of $r<17.7$. Therefore, while we can probe dwarf galaxies with stellar masses comparable to the Magellanic Clouds, we are not sensitive to galaxies with lower stellar mass such as the Fornax dwarf spheroidal $\left(2 \times 10^{7} \mathrm{M}_{\odot}\right)$ around the Milky Way (e.g., McConnachie 2012, Mateo 1998). We also impose modest requirements on emission line

\footnotetext{
3 http://www.nsatlas.org
}

flux measurements reported in the NSA: a signal-to-noise ratio $S / N \geq 3$ and an equivalent width $\mathrm{EW}>1$ for $\mathrm{H} \alpha$, [N II] $\lambda 6584$, and [O III] $\lambda 5007$ and $S / N \geq 2$ for $\mathrm{H} \beta$. This reduces the number of sources to 25,974 .

We use stellar mass as our primary selection criterion rather than absolute magnitude since galaxies of a given mass can span a wide range in absolute magnitude due to differences in stellar populations. Younger, bluer, star-forming galaxies will be brighter than older, redder, quenched galaxies for a given mass (e.g., Bell et al. 2003). Additionally, strong emission lines (and even nebular continuum) can boost broad-band fluxes, especially in the SDSS $g$ - and $r$-bands (e.g., Reines et al. 2010, Anders \& Fritze-v. Alvensleben 2003). Therefore, imposing an absolute magnitude cut would preferentially exclude line-emitting galaxies compared to equivalent galaxies that do not have strong emission lines. The derived stellar masses are less sensitive to these effects since multiple bands are fit and the SED templates of Blanton \& Roweis (2007) include emission lines. However, imposing a stellar mass cut introduces a bias such that blue/starforming galaxies will be detected over a larger volume than red/quenched galaxies with the same stellar mass (e.g., Geha et al. 2012).

\section{ANALYSIS AND RESULTS}

There are many ways to identify the presence of an accreting supermassive BH (see the review in Ho 2008). In this work, we search for two signatures of active BHs in the optical spectra of galaxies: 1) narrow emission-line ratios indicating photoionization by an accreting $\mathrm{BH}$, and 2) broad $\mathrm{H} \alpha$ emission signifying dense gas orbiting a massive BH. After selecting dwarf emission-line galaxies from the NSA ( $\$ 2$, we retrieve the SDSS spectra for the entire sample and analyze them with customized software as described below. All code was developed in the Interactive Data Language (IDL) and our fitting routines make use of the non-linear least squares curve fitting package MPFIT (Markwardt 2009). Individual sources flagged by our automated algorithms are subsequently inspected by eye.

\subsection{Continuum and Absorption Line Subtraction}

Before we can look for any signature of an AGN, we need to model and remove stellar continuum from the host galaxy, that in almost all cases dominates the total continuum. The spectra also contain stellar absorption lines. Balmer absorption is of particular concern since we aim to detect potentially weak broad $\mathrm{H} \alpha$ emission from nuclear activity in the galaxies. Our general approach for modeling the continuum and absorption lines is based on that presented in Tremonti et al. (2004). We fit the galaxy spectra with a non-negative linear combination of simple stellar population (SSP) models spanning a range of ages for a given metallicity. The model templates are the same as those used in Tremonti et al. (2004) and come from the stellar population synthesis code of Bruzual \& Charlot (2003) The templates include model spectra for 10 different ages $(0.005,0.025,0.1,0.29,0.64,0.9$, $1.4,2.5,5$, and $11 \mathrm{Gyr})$ and 3 different metallicities $(Z$ $=0.008,0.02,0.05)$. Each galaxy spectrum is modeled as

4 We retrieved the templates from the corresponding website http://www2.iap.fr/users/charlot/bc2003/ 
a combination of SSP templates with a single metallicity and we select the metallicity yielding the best-fit. In the fitting process, we allow for reddening from dust using the Galactic extinction curve of Cardelli et al. (1989) and Gaussian smoothing to match the absorption line widths.

Our algorithm for modeling the continuum and absorption lines in each spectrum is an iterative process. The SSP models do not contain emission lines and so we begin by masking out pixels $5 \sigma$ above the continuum in the observed spectra to remove both strong and weak lines. We then apply a redshift-correction using the redshift derived from emission lines provided in the NSA catalog. A preliminary model is fit to the resulting spectrum and the two are cross-correlated to determine any redshift offset between the absorption and emission lines. After correcting for the redshift offset, we find the best fit model for each of the 3 model metallicities. The singlemetallicity model with the smallest $\chi^{2}$ value is selected and used to improve the masking of emission lines. Pixels more than $5 \sigma$ above the difference spectrum (data model) are excluded in the last round of fitting. Again, we find the best-fit model for each metallicity and select the single-metallicity model with the smallest $\chi^{2}$ for our final model of the continuum and absorption line spectrum. The vast majority of the galaxy spectra are best fit by the sub-solar metallicity model $(Z=0.008)$, which is consistent with studies demonstrating that low-mass galaxies generally have low metallicities (e.g. Tremonti et al. 2004). In the small fraction of cases where absorption lines are either extremely weak or absent, a thirdorder polynomial is fit to the continuum. We subtract this model from the data to produce a pure-emission line spectrum.

\subsection{Emission Line Measurements}

In order to identify the spectral signatures of active massive BHs, we model the emission lines of interest as Gaussians. We are especially interested in detecting any possible broad $\mathrm{H} \alpha$ component. Luminous unobscured AGN powered by supermassive BHs in massive galaxies often exhibit bright, broad $\mathrm{H} \alpha$ emission that is clearly visible even by eye. However, the broad $\mathrm{H} \alpha$ signatures from AGNs with lower-mass BHs hosted by dwarf galaxies will be less pronounced, generally having narrower widths and lower luminosities than their higher mass counterparts. Moreover, any potential broad $\mathrm{H} \alpha$ feature will be blended with narrow $\mathrm{H} \alpha$ emission and the surrounding [N II] $\lambda \lambda 6548,6583$ doublet. Therefore, it is vital to carefully model the narrow emission line profile in order to detect any potential broad $\mathrm{H} \alpha$ emission.

We base our model of the narrow emission line profile on the [S II] $\lambda \lambda 6713,6731$ doublet, which has been shown to be generally well-matched to the line profiles of the [N II] $\lambda \lambda 6548,6583$ doublet and narrow $\mathrm{H} \alpha$ (Filippenko \& Sargent 1988, 1989; Ho et al. 1997, Greene \& Ho 2004). The [S II] doublet is first fit with a single Gaussian model with the width of the two lines assumed to be equal and the relative separation between the two lines held fixed by their laboratory wavelengths. The [S II] doublet is also fit with a two-component Gaussian model, with the added constraint that the height ratio of the two components must be equal for each line. If the reduced $\chi^{2}$ value from the two-component model is at least $10 \%$ lower than that of the single Gaussian model, the two-component model is selected. Nearly all of the galaxies in our dwarf sample only require a single Gaussian to model the [S II] doublet, with only 15 sources preferring a two-component model ( 8 of which turn out to be classified as either a BPT AGN or composite, see Section 3.3.

Once we have constructed a suitable model of the [S II] doublet, we use it as a template for fitting the narrow lines in the $\mathrm{H} \alpha+[\mathrm{N}$ II $]$ complex. The relative separations between the centroids of the narrow Gaussian components are held fixed using laboratory wavelengths and the flux of [N II] $\lambda 6584$ to [N II] $\lambda 6548$ is fixed at the theoretical value of 2.96. We assume the [N II] lines have the same width (in velocity) as the $[\mathrm{S} \mathrm{II}]$ lines, but generously allow the width of the narrow $\mathrm{H} \alpha$ component to increase by as much as $25 \%$ for the vast majority of cases in which a single Gaussian is used to model the narrow line profile. For the small number of sources $(15 / 25974)$ with two-component Gaussian models for the narrow lines, the profiles are strictly scaled from the [S II] lines. The $\mathrm{H} \alpha+$ $\left[\mathrm{N}\right.$ II] complex is fit and the reduced $\chi^{2}$ computed. The $\mathrm{H} \alpha+[\mathrm{N} \mathrm{II}]$ complex is fit a second time, allowing for an additional broad $\mathrm{H} \alpha$ component. If the resulting reduced $\chi^{2}$ is improved by at least $20 \%$ and the FWHM of the broad $\mathrm{H} \alpha$ component is at least $500 \mathrm{~km} \mathrm{~s}^{-1}$ after correcting for the fiber-dependent instrumental resolution, we select the model including broad $\mathrm{H} \alpha$. Our choice of $20 \%$ improvement in reduced $\chi^{2}$, while somewhat arbitrary, has been used successfully in previous studies (e.g., Hao et al. 2005) and empirically works here. The modest FWHM requirement is imposed to avoid severe contamination from galaxies undergoing intense star formation that have moderately broadened bases on $\mathrm{H} \alpha$. The procedures described thus far yields a sample of 51 sources flagged as having a broad $\mathrm{H} \alpha$ component, not all of which make it into our final sample of broad-line AGN candidates as described in Section 3.4

We also measure the $\mathrm{H} \beta$, [O III] $\lambda 5007$ and [O I] 6300 emission lines. The narrow-line profile derived from the [S II] doublet is used as a template for fitting $\mathrm{H} \beta$, using the same approach as that for $\mathrm{H} \alpha$. The $\mathrm{H} \beta$ line is fit twice, with and without a broad component. If statistically justified (reduced $\chi^{2}$ is improved by at least $20 \%$ ), we accept the model with a broad component. Since the [O III] profile commonly exhibits a broad, blue shoulder (e.g., Heckman et al. 1981, Whittle 1985) and does not typically match the profile of the other narrow lines measured in this work (Greene \& Ho 2005a), we do not use the model derived from the $[\mathrm{S}$ II $]$ doublet. Instead, we fit the [O III] (and [O I]) line independently allowing for up to two Gaussian components ( $\chi^{2}$ must be reduced by at least $20 \%$ to use the two-component model).

Emission-line fluxes are calculated using the Gaussian model parameters. Uncertainties in the model parameters are provided by MPFIT, which accounts for the SDSS error spectrum. We use standard propagation of errors to determine the errors in the line fluxes.

\subsection{BPT AGN and Composites}

When an accreting $\mathrm{BH}$ is present in a galaxy, the ISM is photoionized by a much harder continuum than is emitted by hot stars. AGNs and H II regions separate cleanly in two-dimensional strong line diagnostic diagrams that take pairs of lines close together in frequency to mitigate 

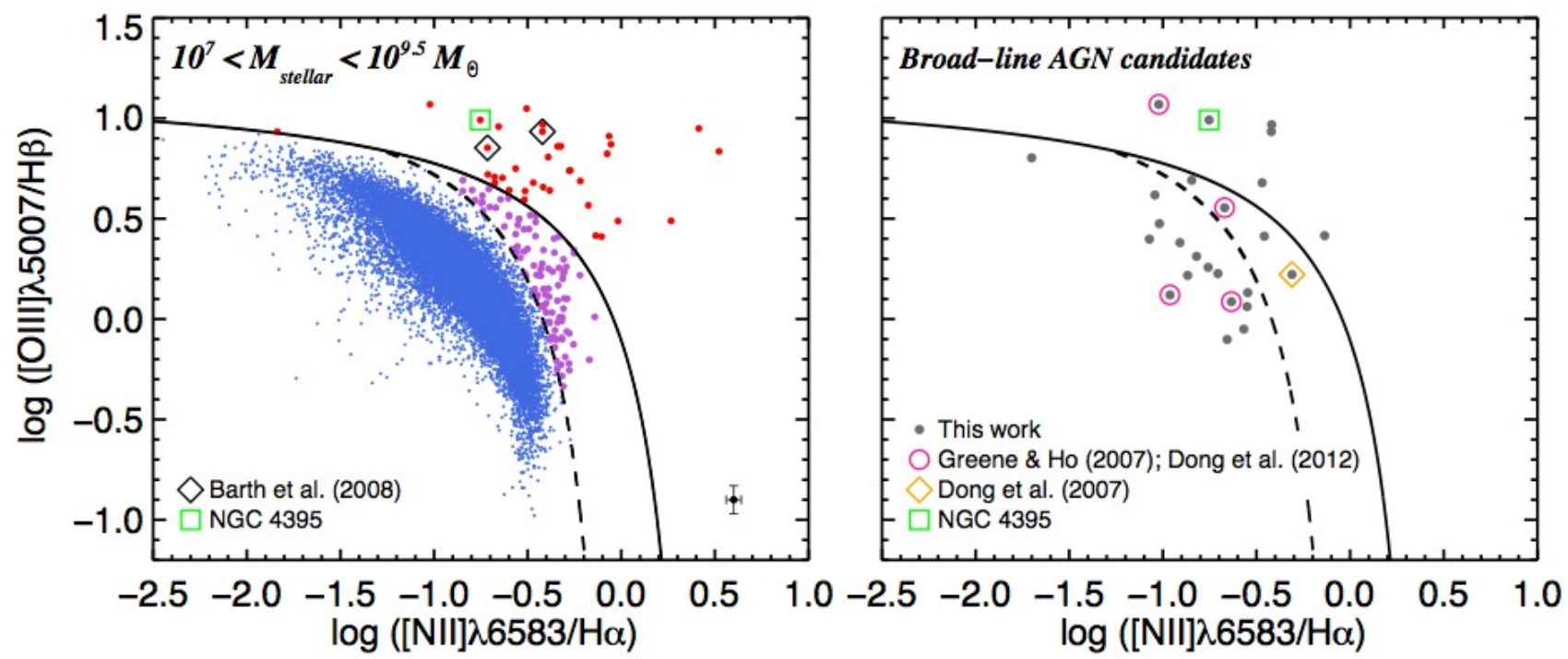

Figure 1. Left: BPT [O III] $/ \mathrm{H} \beta$ versus $[\mathrm{N} \mathrm{II}] / \mathrm{H} \alpha$ narrow-line diagnostic diagram for all $\sim 25,000$ dwarf emission-line galaxies analyzed in this work. Galaxies with spectra dominated by an AGN are plotted as red points and galaxies with spectra dominated by star formation are plotted as blue points. Composite galaxies with significant contributions from both an AGN and star formation are plotted as purple points. The typical error is shown in the lower right corner (individual flux errors are given in Tables 2 and 40. The dashed line is an empirical separation of pure star-forming galaxies and those with some contribution from an AGN from Kaufmann et al. (2003b). The solid line is from Kewley et al. (2001), indicating the 'maximum starburst line' given by pure stellar photolonization models. We note that the red point falling to the far lett of the diagram and just above the dividing line is unusual in a number of ways and rather suspect (see footnote in text). Right: BPT diagram for broad-line AGN candidates only (\$3.4). We consider the sources falling in the AGN and composite regions of the diagram the most secure broad-line candidates. Significant contamination from luminous Type II supernovae is likely in the star-forming region of the diagram.

the effects of reddening (Baldwin et al. 1981; Veilleux \& Osterbrock 1987, Kewley et al. 2001; Kauftmann et al. 2003b; Kewley et al. 2006). The two-dimensional linediagnostic diagrams (also known as BPT diagrams after the Baldwin et al. paper) are routinely used to separate line-emitting galaxies by their primary excitation source.

Here we employ the most widely used BPT diagram as our primary diagnostic, which takes $\left[\begin{array}{ll}\mathrm{O} & \mathrm{III}\end{array}\right] / \mathrm{H} \beta$ vs. $\left[\begin{array}{ll}\mathrm{N} & \mathrm{II}\end{array}\right] / \mathrm{H} \alpha$ (Figure 1 ). In this diagram, line-emitting galaxies separate into a $V$-shape (e.g., Kewley et al. 2006: Groves et al. 2006). Star-forming galaxies with H II-region-like spectra occupy the left-most plume of galaxies, with lower-metallicity systems having higher $[\mathrm{O} \mathrm{III}] / \mathrm{H} \beta$ ratios and lower $[\mathrm{N} \mathrm{II}] / \mathrm{H} \alpha$ ratios (e.g., Moustakas et al. 2006). AGNs occupy the right branch of galaxies with high-ionization Seyferts found in the upper right. Low-metallicity AGNs are found to the left of the main Seyfert region and can overlap with lowmetallicity starburst galaxies (Groves et al. 2006, Ludwig et al. 2012). The most contentious region of the diagram sits directly below the Seyfert galaxies: galaxies with very high low-ionization lines, named Low Ionization Nuclear Emission Region galaxies by Heckman (LINERs; 1980). LINER emission can be generated both by shocks and very hard AGN spectra, and disentangling the primary origin in any given case can be complicated and aperture dependent (e.g., Kewley et al. 2006; Sarzi et al. 2006, Ho 2008; Eracleous et al. 2010, Yan \& Blanton 2012). Finally, composites objects fall in the region delineated by the empirical dividing line of Kauffmann et al. (2003b) separating pure star-forming galaxies from those with some contribution from an AGN, and the theoretical 'maximum starburst line' of Kewley et al. (2001) given by pure stellar photoionization models. Composite objects are thought to contain significant contributions from both AGN and star formation (e.g., Panessa et al. 2005; Kewley et al. 2006; Trouille et al. 2011, but also see Liu et al. (2008)).

U sing the $[\mathrm{O}$ III $] / \mathrm{H} \beta$ vs. $[\mathrm{N} \mathrm{II}] / \mathrm{H} \alpha$ diagnostic diagram and our narrow emission-line measurements, we have identified 136 galaxies with photoionization signatures of an active massive $\mathrm{BH}$ (Figure 1, Tables 1 and 2). There are 35 galaxies with narrow-line ratios falling in the AGN-dominated region of the BPT diagram and 101 galaxies with composite spectra. While we consider the BPT-AGN more secure ${ }^{5}$, the composite spectra likely indicate at least some contribution from an AGN (Trouille et al. 2011; Jia et al. 2011), which for our purposes is sufficient for tentatively identifying the presence of a massive BH. Even with our inclusive approach, only $\sim 0.5 \%(136 / 25974)$ of dwarf emission-line galaxies in our parent sample exhibit optical narrow-line signatures of photoionization from an accreting $\mathrm{BH}$ (although see the discussion in Section 5 for a number of caveats).

We also investigate the positions of AGNs and composite galaxies according to the $[\mathrm{O}$ III $] / \mathrm{H} \beta$ vs. $[\mathrm{N} \mathrm{II}] / \mathrm{H} \alpha$ diagram in two secondary diagnostic diagrams, $[\mathrm{O}$ III $] / \mathrm{H} \beta$ vs. $[\mathrm{S} \quad \mathrm{II}] / \mathrm{H} \alpha$ and $[\mathrm{O} \quad \mathrm{III}] / \mathrm{H} \beta$ vs. $\left[\begin{array}{ll}\mathrm{O} & \mathrm{I}\end{array}\right] / \mathrm{H} \alpha$ (Figure 2 ). $\left[\begin{array}{ll}\mathrm{O} & \mathrm{I}\end{array}\right] / \mathrm{H} \alpha$ is particularly useful as it is sensitive to the hardness of the ionizing radiation field (e.g., Kewley et al.

\footnotetext{
5 The left-most red point in Figure 1 corresponds to a bright offnuclear source in a blue late-type irregular dwarf galaxy, that may in fact be an extreme H II region. While we cannot exclude the possibility that this source is a low-metallicity active massive $\mathrm{BH}$, we consider it rather suspect in the absence of other supporting evidence.
} 
Table 1

BPT AGN and Composites: Galaxy Properties

\begin{tabular}{|c|c|c|c|c|c|c|c|c|c|}
\hline $\begin{array}{l}\text { ID } \\
(1)\end{array}$ & $\begin{array}{l}\text { NSAID } \\
(2)\end{array}$ & $\begin{array}{c}\text { SDSS Name } \\
\text { (3) }\end{array}$ & $\begin{array}{c}\text { Plate-MJD-Fiber } \\
(4)\end{array}$ & $\begin{array}{c}z \\
(5)\end{array}$ & $\underset{(6)}{\log M_{\star}}$ & $\begin{array}{l}M_{g} \\
(7)\end{array}$ & $\begin{array}{c}g-r \\
(8)\end{array}$ & $\begin{array}{l}r_{50} \\
(9)\end{array}$ & $\begin{array}{l}\text { Sérsic } n \\
\quad(10)\end{array}$ \\
\hline \multicolumn{10}{|c|}{$A G N s$} \\
\hline 1 & 62996 & J024656.39-003304.8 & $1561-53032-161$ & 0.0459 & 9.41 & -17.99 & 0.81 & 1.52 & 1.0 \\
\hline 2 & 7480 & J024825.26-002541.4 & 409-51871-150 & 0.0247 & 9.11 & -17.32 & 0.58 & 1.43 & 2.0 \\
\hline 3 & 68765 & J032224.64+401119.8 & $1666-52991-48$ & 0.0261 & 9.42 & -18.65 & 0.47 & 0.93 & 3.5 \\
\hline 4 & 64339 & J081145.29+232825.7 & $1584-52943-567$ & 0.0157 & 9.02 & -17.98 & 0.36 & 0.61 & 4.4 \\
\hline 5 & 46677 & J082334.84+031315.6 & $1185-52642-123$ & 0.0098 & 8.54 & -18.85 & -0.29 & 1.66 & 2.8 \\
\hline 6 & 105376 & J084025.54+181858.9 & $2278-53711-445$ & 0.0150 & 9.28 & -17.61 & 0.59 & 1.00 & 3.8 \\
\hline 7 & 30020 & J084204.92+403934.5 & $829-52296-391$ & 0.0293 & 9.30 & -17.45 & 0.62 & 1.32 & 1.6 \\
\hline 8 & 110040 & J090222.76+141049.2 & $2433-53820-569$ & 0.0297 & 9.41 & -17.91 & 0.59 & 1.36 & 1.4 \\
\hline 9 & 10779 & J090613.75+561015.5 & $450-51908-409$ & 0.0466 & 9.36 & -18.98 & 0.40 & 1.63 & 4.1 \\
\hline 10 & 106134 & J092129.98+213139.3 & $2290-53727-185$ & 0.0313 & 9.31 & -18.20 & 0.58 & 1.07 & 6.0 \\
\hline 11 & 125318 & J095418.15+471725.1 & $2956-54525-457$ & 0.0327 & 9.12 & -18.73 & 0.44 & 2.00 & 6.0 \\
\hline 12 & 107272 & $\mathrm{~J} 100935.66+265648.9$ & $2347-53757-456$ & 0.0144 & 8.44 & -17.56 & 0.31 & 0.66 & 6.0 \\
\hline 13 & 37942 & $\mathrm{~J} 102252.21+464220.7$ & $961-52615-346$ & 0.0400 & 9.44 & -18.08 & 0.66 & 1.05 & 5.8 \\
\hline 14 & 111644 & $\mathrm{~J} 110503.96+224123.4$ & $2488-54149-561$ & 0.0246 & 9.05 & -16.91 & 0.74 & 0.61 & 6.0 \\
\hline $15^{\mathrm{a}}$ & 27397 & $\mathrm{~J} 110912.37+612347.0$ & $774-52286-554$ & 0.0068 & 8.91 & -17.33 & 0.36 & 1.59 & 1.2 \\
\hline 16 & 30370 & $\mathrm{~J} 111319.23+044425.1$ & $835-52326-433$ & 0.0265 & 9.28 & -17.98 & 0.49 & 1.31 & 4.3 \\
\hline 17 & 101949 & $\mathrm{~J} 114302.40+260818.9$ & $2221-53792-218$ & 0.0230 & 9.26 & -17.39 & 0.59 & 0.74 & 1.9 \\
\hline 18 & 113566 & J114359.58+244251.7 & $2510-53877-255$ & 0.0501 & 9.48 & -18.68 & 0.65 & 1.32 & 5.6 \\
\hline 19 & 92369 & $\mathrm{~J} 114418.83+334007.4$ & $2097-53491-228$ & 0.0325 & 9.38 & -17.76 & 0.62 & 0.87 & 6.0 \\
\hline $20^{\mathrm{b}}$ & 52675 & $\mathrm{~J} 122342.82+581446.4$ & $1315-52791-568$ & 0.0144 & 9.47 & -18.11 & 0.66 & 1.04 & 5.7 \\
\hline $21^{\mathrm{c}}$ & 89394 & J122548.86+333248.7 & $2015-53819-251$ & 0.0011 & 9.10 & -18.05 & 0.35 & 1.71 & 1.7 \\
\hline 22 & 77431 & $\mathrm{~J} 130434.92+075505.0$ & $1794-54504-547$ & 0.0480 & 8.95 & -18.77 & 0.45 & 1.23 & 5.6 \\
\hline 23 & 89494 & $\mathrm{~J} 130457.86+362622.2$ & $2016-53799-414$ & 0.0229 & 9.47 & -18.64 & 0.45 & 3.94 & 0.8 \\
\hline 24 & 104527 & $\mathrm{~J} 133245.62+263449.3$ & $2245-54208-467$ & 0.0470 & 9.39 & -19.07 & 0.27 & 1.18 & 2.3 \\
\hline 25 & 51220 & $\mathrm{~J} 134757.69+465434.9$ & $1284-52736-95$ & 0.0277 & 9.37 & -18.13 & 0.55 & 0.62 & 6.0 \\
\hline 26 & 54572 & $\mathrm{~J} 134939.36+420241.4$ & $1345-52814-41$ & 0.0411 & 9.33 & -18.55 & 0.47 & 0.89 & 3.0 \\
\hline 27 & 78568 & $\mathrm{~J} 140228.72+091856.4$ & $1807-54175-560$ & 0.0191 & 8.93 & -16.57 & 0.69 & 0.87 & 1.6 \\
\hline 28 & 70907 & $\mathrm{~J} 140510.39+114616.9$ & $1703-53799-510$ & 0.0174 & 9.37 & -18.52 & 0.42 & 1.30 & 4.5 \\
\hline 29 & 71023 & $\mathrm{~J} 141208.47+102953.8$ & $1705-53848-98$ & 0.0326 & 9.08 & -17.74 & 0.42 & 0.62 & 3.9 \\
\hline 30 & 123656 & $\mathrm{~J} 142044.94+224236.8$ & $2786-54540-552$ & 0.0307 & 9.27 & -18.35 & 0.58 & 1.07 & 5.9 \\
\hline 31 & 71565 & $\mathrm{~J} 143523.42+100704.2$ & $1711-53535-306$ & 0.0312 & 9.24 & -18.53 & 0.57 & 0.97 & 2.9 \\
\hline $32^{\mathrm{a}}$ & 15235 & $\mathrm{~J} 144012.70+024743.5$ & $536-52024-575$ & 0.0299 & 9.46 & -19.18 & 0.31 & 1.70 & 2.7 \\
\hline 33 & 120870 & $\mathrm{~J} 144712.80+133939.2$ & $2750-54242-275$ & 0.0323 & 9.32 & -18.31 & 0.53 & 1.54 & 1.8 \\
\hline 34 & 124249 & $\mathrm{~J} 153941.68+171421.8$ & 2795-54563-509 & 0.0458 & 9.45 & -18.99 & 0.39 & 1.19 & 3.6 \\
\hline 35 & 64022 & $\mathrm{~J} 154059.61+315507.3$ & $1581-53149-255$ & 0.0528 & 9.12 & -19.36 & 0.17 & 1.33 & 6.0 \\
\hline \multicolumn{10}{|c|}{ Composites } \\
\hline 36 & 5790 & J002145.80+003327.3 & $390-51900-465$ & 0.0178 & 9.16 & -17.05 & 0.65 & 0.90 & 1.1 \\
\hline 37 & 6059 & J010005.93-011058.8 & $395-51783-58$ & 0.0514 & 9.34 & -18.76 & 0.59 & 1.19 & 5.7 \\
\hline 38 & 24013 & J024635.03+000719.1 & $707-52177-494$ & 0.0286 & 9.06 & -17.48 & 0.50 & 1.21 & 1.0 \\
\hline 39 & 24061 & J030644.60-002431.5 & $710-52203-279$ & 0.0252 & 9.41 & -18.16 & 0.51 & 1.18 & 0.9 \\
\hline 40 & 82616 & J074829.21+510052.4 & $1869-53327-263$ & 0.0190 & 9.10 & -17.89 & 0.38 & 3.05 & 1.7 \\
\hline 41 & 83933 & J080228.84+203050.3 & $1922-53315-364$ & 0.0286 & 8.60 & -19.85 & 0.78 & 2.04 & 2.0 \\
\hline 42 & 75073 & J081010.69+073337.1 & $1756-53080-413$ & 0.0522 & 9.37 & -19.53 & 0.42 & 1.88 & 6.0 \\
\hline 43 & 31779 & J081353.45+310824.3 & $861-52318-72$ & 0.0479 & 9.41 & -18.77 & 0.72 & 1.20 & 6.0 \\
\hline 44 & 50121 & J081549.08+254701.4 & $1266-52709-127$ & 0.0248 & 9.18 & -17.21 & 0.63 & 0.87 & 2.1 \\
\hline 45 & 35979 & J082013.92+302503.0 & $931-52619-145$ & 0.0196 & 9.43 & -17.97 & 0.59 & 1.12 & 1.7 \\
\hline 46 & 83280 & J083740.88+601339.3 & $1874-54452-158$ & 0.0273 & 9.45 & -18.72 & 0.46 & 2.00 & 0.5 \\
\hline 47 & 64526 & J084449.13+281853.5 & $1588-52965-176$ & 0.0205 & 9.45 & -17.84 & 0.59 & 0.93 & 2.5 \\
\hline 48 & 47066 & J085125.81+393541.7 & $1198-52669-392$ & 0.0410 & 9.41 & -19.19 & 0.28 & 1.76 & 0.5 \\
\hline 49 & 36154 & J085548.25+354502.0 & $935-52643-53$ & 0.0527 & 9.45 & -19.19 & 0.42 & 5.62 & 1.7 \\
\hline 50 & 47918 & J090737.05+352828.4 & $1212-52703-97$ & 0.0276 & 9.44 & -18.53 & 0.53 & 1.17 & 5.4 \\
\hline
\end{tabular}

Note. - Table 1 is published in its entirety in the electronic edition of The Astrophysical Journal. A portion is shown here for guidance regarding its form and content. Col.(1): Identification number assigned in this paper. Col.(2): NSA identification number. Col.(3): SDSS name. Col(4): Plate-MJD-Fiber of analyzed spectra. Col.(5): Redshift. Col.(6): Log galaxy stellar mass in units of M $\odot$. Col.(7): Absolute $g$-band magnitude. Col.(8): $g-r$ color. Col.(9): Petrosian 50\% light radius in units of kpc. Col.(10): Sérsic index, $n$. All values are from the NSA and assume $h=0.73$ Magnitudes are $K$-corrected to rest-frame values using kcorrect v4_2 and corrected for foreground Galactic extinction.

a Galaxies in Barth et al. (2008)

b Galaxies in Greene \& Ho (2007) and Dong et al. (2012)

c NGC 4395 Filippenko \& Sargent 1989 Filippenko \& Ho 2003

d Galaxy in Dong et al. (2007) 
Table 2

BPT AGN and Composites: Emission Line Fluxes

\begin{tabular}{|c|c|c|c|c|c|c|c|c|c|c|}
\hline $\begin{array}{l}\text { ID } \\
(1)\end{array}$ & $\begin{array}{l}(\mathrm{H} \beta)_{n} \\
\quad(2)\end{array}$ & $\begin{array}{c}(\mathrm{H} \beta)_{b} \\
(3)\end{array}$ & $\begin{array}{c}{[\mathrm{O} \text { III }] \lambda 5007} \\
(4)\end{array}$ & 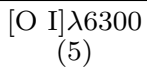 & $\begin{array}{c}{[\mathrm{N} \text { II] }] \lambda 6548} \\
(6)\end{array}$ & $\begin{array}{c}(\mathrm{H} \alpha)_{n} \\
\quad(7)\end{array}$ & $\begin{array}{c}(\mathrm{H} \alpha)_{b} \\
(8)\end{array}$ & $\begin{array}{c}{[\mathrm{N} \text { II }] \lambda 6583} \\
(9)\end{array}$ & $\begin{array}{c}{[\mathrm{S} \text { II }] \lambda 6716} \\
(10)\end{array}$ & $\begin{array}{c}{\left[\begin{array}{ll}\mathrm{S} & \mathrm{II}\end{array}\right] \lambda 6731} \\
(11)\end{array}$ \\
\hline \multicolumn{11}{|c|}{$A G N s$} \\
\hline 1 & $11(3)$ & $\cdots$ & $29(3)$ & $\cdots$ & $8(1)$ & $34(6)$ & $56(9)$ & $25(4)$ & $8(2)$ & $6(1)$ \\
\hline 2 & $13(3)$ & $\ldots$ & $52(5)$ & $\ldots$ & $6(1)$ & $61(3)$ & $\ldots$ & $19(3)$ & $14(3)$ & $11(2)$ \\
\hline 3 & $100(11)$ & $\ldots$ & $1119(61)$ & $45(3)$ & $44(5)$ & $418(45)$ & $\ldots$ & $130(6)$ & $64(8)$ & $67(11)$ \\
\hline 4 & $82(6)$ & $\ldots$ & $460(75)$ & $57(6)$ & $28(2)$ & $299(7)$ & $\ldots$ & $82(5)$ & $74(5)$ & $68(4)$ \\
\hline 5 & $5813(69)$ & $\ldots$ & 49827(429) & $144(6)$ & $100(2)$ & 20342(193) & $\ldots$ & $296(5)$ & $520(9)$ & $420(6)$ \\
\hline 6 & $101(6)$ & $\ldots$ & $515(11)$ & $20(4)$ & $22(1)$ & $313(8)$ & $\ldots$ & $66(4)$ & $71(5)$ & $50(3)$ \\
\hline 7 & $13(2)$ & $\ldots$ & $62(4)$ & & $13(1)$ & $62(5)$ & $\ldots$ & $38(4)$ & $25(3)$ & $20(2)$ \\
\hline 8 & 19(3) & $\ldots$ & $58(15)$ & $13(4)$ & $29(2)$ & $90(7)$ & $\ldots$ & $87(7)$ & $43(5)$ & $31(3)$ \\
\hline 9 & 151(13) & $79(12)$ & $1398(34)$ & 121(10) & $68(5)$ & $530(42)$ & $322(14)$ & 202(10) & $97(9)$ & $79(13)$ \\
\hline 10 & $34(3)$ & $\ldots$ & $155(6)$ & & $17(1)$ & $131(5)$ & & $50(3)$ & $47(4)$ & $30(2)$ \\
\hline 11 & $510(20)$ & $\ldots$ & $2433(44)$ & 179(10) & $188(7)$ & $1643(66)$ & $118(16)$ & $557(18)$ & $267(16)$ & $251(26)$ \\
\hline 12 & $336(8)$ & $\ldots$ & $3048(87)$ & $226(6)$ & $90(2)$ & $1199(16)$ & & $265(5)$ & $233(5)$ & $182(4)$ \\
\hline 13 & $22(4)$ & $\ldots$ & $56(6)$ & $\ldots$ & $20(2)$ & $77(7)$ & $\cdots$ & $60(6)$ & $45(6)$ & $33(4)$ \\
\hline 14 & $15(3)$ & $\ldots$ & $109(6)$ & & $10(1)$ & $63(5)$ & $\ldots$ & $30(4)$ & $21(4)$ & $13(2)$ \\
\hline 15 & $93(5)$ & $\ldots$ & $660(11)$ & $40(3)$ & $21(1)$ & $327(6)$ & $\ldots$ & $63(2)$ & $87(3)$ & $72(2)$ \\
\hline 16 & $33(4)$ & $\ldots$ & $171(6)$ & & $8(1)$ & $128(4)$ & $\ldots$ & $25(2)$ & $34(3)$ & $22(2)$ \\
\hline 17 & $125(9)$ & $\ldots$ & $459(13)$ & $47(6)$ & $121(6)$ & $535(12)$ & $\cdots$ & $357(17)$ & $84(6)$ & $83(4)$ \\
\hline 18 & $22(4)$ & $\ldots$ & $120(23)$ & & $17(2)$ & $94(5)$ & $\ldots$ & $50(6)$ & $23(4)$ & $16(2)$ \\
\hline 19 & $23(6)$ & $\ldots$ & $154(18)$ & $27(5)$ & $33(3)$ & $117(11)$ & $\ldots$ & $98(9)$ & $43(6)$ & $32(4)$ \\
\hline 20 & 211(13) & $495(37)$ & $2477(49)$ & $120(10)$ & $52(4)$ & $1604(82)$ & $3195(50)$ & $153(11)$ & $98(7)$ & $101(6)$ \\
\hline 21 & $3314(47)$ & $893(39)$ & $32481(394)$ & $3542(49)$ & $777(11)$ & 13026(162) & $6167(73)$ & $2300(30)$ & $2434(35)$ & 2994(59) \\
\hline 22 & $16(3)$ & $\ldots$ & $140(6)$ & & $42(4)$ & $48(4)$ & $\ldots$ & $125(12)$ & $29(4)$ & $18(2)$ \\
\hline 23 & $39(5)$ & $\ldots$ & $215(7)$ & $18(3)$ & $23(1)$ & $128(4)$ & $\ldots$ & $68(4)$ & $52(4)$ & $37(2)$ \\
\hline 24 & $539(22)$ & $\ldots$ & $3905(67)$ & $238(15)$ & $317(13)$ & $2048(83)$ & $\ldots$ & $937(27)$ & 394(18) & $367(22)$ \\
\hline 25 & $151(9)$ & $\ldots$ & 1034(183) & $78(8)$ & $671(21)$ & $597(13)$ & $\ldots$ & $1985(63)$ & 165(8) & $124(5)$ \\
\hline 26 & $93(8)$ & $\ldots$ & 691(16) & $26(7)$ & $102(5)$ & $344(10)$ & $\ldots$ & $303(16)$ & $74(6)$ & $61(4)$ \\
\hline 27 & $32(4)$ & $\ldots$ & $202(6)$ & $26(3)$ & $22(1)$ & $158(4)$ & $\ldots$ & $64(3)$ & $53(3)$ & $41(2)$ \\
\hline 28 & $81(7)$ & $\ldots$ & $353(11)$ & $56(7)$ & $42(2)$ & $299(9)$ & $\ldots$ & $124(7)$ & $102(7)$ & $73(4)$ \\
\hline 29 & $63(6)$ & $\ldots$ & $516(12)$ & $55(5)$ & $82(3)$ & $280(7)$ & $\cdots$ & 241(10) & $82(5)$ & $64(4)$ \\
\hline 30 & $33(5)$ & $\ldots$ & $142(6)$ & & $9(1)$ & $87(4)$ & $\ldots$ & $27(4)$ & $21(4)$ & $21(3)$ \\
\hline 31 & $156(8)$ & $\ldots$ & $682(33)$ & $30(8)$ & $49(3)$ & $581(12)$ & $\ldots$ & $146(10)$ & $62(6)$ & $51(4)$ \\
\hline 32 & $832(19)$ & $132(15)$ & $7136(94)$ & $375(13)$ & $448(8)$ & $3496(66)$ & $301(36)$ & $1326(19)$ & $502(11)$ & $495(15)$ \\
\hline 33 & $23(3)$ & $\ldots$ & $72(6)$ & $18(6)$ & $49(4)$ & $79(7)$ & $\ldots$ & $145(12)$ & $53(5)$ & $42(4)$ \\
\hline 34 & $180(6)$ & $\ldots$ & $906(38)$ & $15(4)$ & $47(2)$ & $597(10)$ & $\ldots$ & $138(5)$ & $90(4)$ & $66(3)$ \\
\hline 35 & $470(20)$ & $\ldots$ & $2233(90)$ & $90(5)$ & $118(5)$ & $1661(64)$ & $\ldots$ & $351(8)$ & $310(13)$ & $228(12)$ \\
\hline \multicolumn{11}{|c|}{ Composites } \\
\hline 36 & $52(4)$ & $\cdots$ & $115(4)$ & $27(3)$ & $40(1)$ & $212(4)$ & $\cdots$ & $119(4)$ & $67(3)$ & $49(2)$ \\
\hline 37 & $299(23)$ & $\ldots$ & $878(101)$ & $64(5)$ & $105(8)$ & $1113(83)$ & $\ldots$ & $312(16)$ & $216(26)$ & $160(33)$ \\
\hline 38 & $14(3)$ & $\ldots$ & $18(4)$ & $\ldots$ & $5(1)$ & $43(6)$ & $\cdots$ & $15(2)$ & $15(3)$ & $11(2)$ \\
\hline 39 & $35(4)$ & $\ldots$ & $40(5)$ & $\ldots$ & $17(1)$ & $134(5)$ & $\ldots$ & $51(4)$ & $34(3)$ & $28(3)$ \\
\hline 40 & $19(3)$ & $\ldots$ & $86(5)$ & $6(2)$ & $5(1)$ & $82(4)$ & $\ldots$ & $16(2)$ & $20(3)$ & $13(2)$ \\
\hline 41 & $83(8)$ & $\ldots$ & $71(10)$ & & $65(5)$ & $362(12)$ & $\ldots$ & $191(15)$ & $88(9)$ & $53(6)$ \\
\hline 42 & $327(44)$ & $\ldots$ & $618(47)$ & $18(5)$ & $161(22)$ & 1260(168) & $\cdots$ & $477(45)$ & 102(18) & $98(30)$ \\
\hline 43 & $41(5)$ & $\ldots$ & $36(5)$ & & $20(2)$ & 133(6) & $\ldots$ & $59(5)$ & $36(4)$ & $22(3)$ \\
\hline 44 & $109(14)$ & $\ldots$ & $118(9)$ & $38(6)$ & $66(8)$ & $495(59)$ & $\ldots$ & $197(35)$ & $140(26)$ & $106(34)$ \\
\hline 45 & $18(5)$ & $\ldots$ & $27(6)$ & & $9(1)$ & $61(5)$ & $\ldots$ & $28(4)$ & $22(4)$ & $25(4)$ \\
\hline 46 & $24(4)$ & $\cdots$ & $13(4)$ & $\ldots$ & $15(1)$ & $97(4)$ & $\cdots$ & $44(4)$ & $26(3)$ & $15(2)$ \\
\hline 47 & $27(4)$ & $\ldots$ & $32(5)$ & $16(4)$ & $18(1)$ & $133(5)$ & $\ldots$ & $53(4)$ & $38(4)$ & $22(3)$ \\
\hline 48 & $169(7)$ & $\ldots$ & $438(10)$ & $31(5)$ & $61(2)$ & $518(11)$ & $137(18)$ & $180(6)$ & $110(5)$ & $75(3)$ \\
\hline 49 & $8(3)$ & $\ldots$ & $18(4)$ & 0() & $4(1)$ & $48(3)$ & $\ldots$ & $13(2)$ & $16(2)$ & $10(1)$ \\
\hline 50 & $107(7)$ & $\cdots$ & $350(10)$ & $25(6)$ & $42(2)$ & $358(9)$ & $\cdots$ & $125(6)$ & $93(6)$ & $73(4)$ \\
\hline
\end{tabular}

Note. - Table 2 is published in its entirety in the electronic edition of The Astrophysical Journal. A portion is shown here for guidance regarding its form and content. Col.(1): Identification number assigned in this paper. Col.(2)-(11): Emission line fluxes with units of $10^{-17} \mathrm{erg} \mathrm{s}^{-1} \mathrm{~cm}-2$. Errors are shown in parenthesis. No line is detected. In the cases where a broad component of $\mathrm{H} \alpha$ or $\mathrm{H} \beta$ is detected, we use the flux of the narrow component to calculate the emission line ratios for the BPT diagram. 
2006), although [O I] is relatively weak and not detected in $\sim 50 \%$ of the AGNs + composites (Table 2). Figure 2 shows that nearly all of the $[\mathrm{N} \mathrm{II}] / \mathrm{H} \alpha$ AGN fall in the Seyfert region of the secondary diagnostic diagrams, with at most 3 falling in the LINER part of the diagrams. The $[\mathrm{N}$ II $] / \mathrm{H} \alpha$ composites fall throughout the $[\mathrm{S}$ II $] / \mathrm{H} \alpha$ and $[\mathrm{O}$ I $] / \mathrm{H} \alpha$ diagrams.

The SDSS spectra of the BPT-AGN are shown in Figures 3 and 4 with the continuum and absorption line models over-plotted in blue. Our sample of BPT-AGN includes NGC 4395 and 2 galaxies from the low-mass Seyfert 2 sample of Barth et al. (2008). The remaining galaxies in Barth et al. (2008) do not meet the selection criteria to be included in our parent dwarf galaxy sample (\$2). Due to the large number of composites, we do not show their spectra here. However, we have inspected all of the individual spectra flagged as composites by eye and cut sources with unreliable emission line measurements due to low signal-to-noise. Twenty objects were excluded, leaving a final sample of 101 composites.

\subsection{Broad-Line AGN Candidates}

We also search for broad $\mathrm{H} \alpha$ emission in our parent sample of galaxies, which may indicate dense gas orbiting a $\mathrm{BH}$ within the broad-line region (BLR), only light-days from the central BH (e.g., Peterson et al. 2004 2005. Bentz et al. 2009b). Unobscured quasars powered by $\sim 10^{8} \mathrm{M}_{\odot}$ BHs have typical line-widths of $\sim 3000 \mathrm{~km}$ $\mathrm{s}^{-1}$ (e.g., Shen et al. 2008). However, in AGNs with lowmass BHs $\left(M_{\mathrm{BH}} \lesssim 10^{6.5} \mathrm{M}_{\odot}\right)$, the line-widths can be just hundreds of $\mathrm{km} \mathrm{s}^{-1}$ (e.g., Filippenko \& Ho 2003 Greene \& Ho 2007a). While in principle broad emission lines provide clear evidence that gas is moving in the potential of a compact massive object (e.g., Ho et al. 1997), there are a few complications, particularly in this lowmass regime. First, $\mathrm{H} \alpha$ absorption from young stars can mask or mimic the broad $\mathrm{H} \alpha$, and thus accurate galaxy continuum subtraction is required (Section 3.1; also see Greene \& Ho 2004). Second, some varieties of supernovae (SNe) can masquerade as AGNs at low-luminosities (see below).

Out of the 51 sources flagged as having a broad $\mathrm{H} \alpha$ component in their spectra with a FWHM $\geq 500 \mathrm{~km}$ $\mathrm{s}^{-1}$ (see 3.2 , 25 make it into our final sample of broadline AGN candidates after a more careful examination of each individual source. We detect broad $\mathrm{H} \beta$, in addition to broad $\mathrm{H} \alpha$ in $36 \%(9 / 25)$ of these sources (Tables 2 and 4). We have excluded 9 likely Type II SNe (most having characteristic $\mathrm{P}$ Cygni profiles), 3 non-nuclear star-forming knots in nearby galaxies (with low broad $\mathrm{H} \alpha$ luminosities), 1 Luminous Blue Variable star identified by Izotov \& Thuan (2009b, NSAID 5109), and 13 sources in the star-forming region of the BPT diagram with marginal detections of broad $\mathrm{H} \alpha$ that are unconvincing by eye. Figure 5 shows the spectral fits for one of our broad-line AGN candidates. Plots for the other broad-line AGN candidates are shown in the Appendix.

Our broad-line sample includes NGC 4395, the dwarf disk galaxy presented in Dong et al. (2007), and 4 galaxies from the samples of Greene \& Ho (2007a) and Dong et al. (2012). The remaining samples of low-mass BHs in the latter two works are hosted in galaxies that do not meet the selection criteria to be included in our
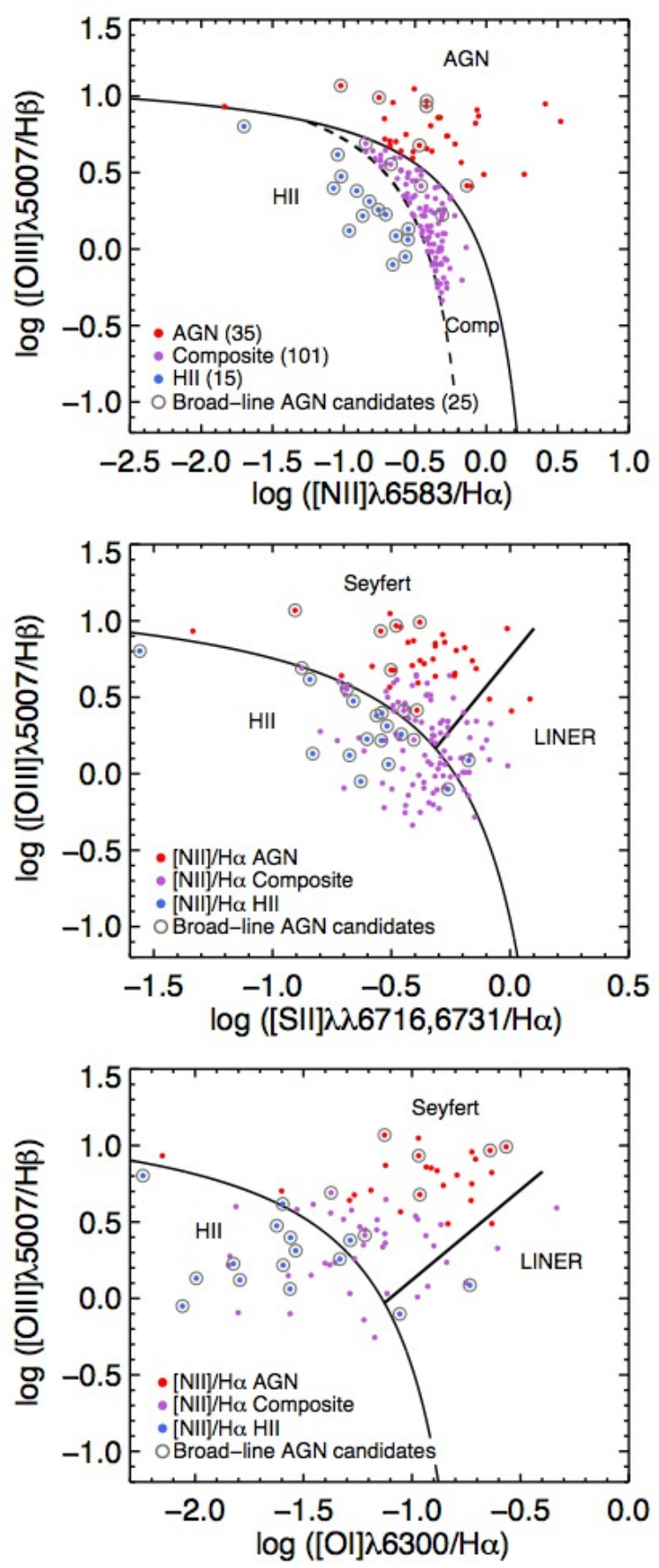

Figure 2. BPT narrow-line diagnostic diagrams for our sample of active galaxies. Regions are delineated according to the classification scheme outlined in Kewley et al. (2006). Top: $[\mathrm{O}$ III $] / \mathrm{H} \beta$ versus $[\mathrm{N} \mathrm{II}] / \mathrm{H} \alpha$ diagram. There are 35 galaxies in the AGN part of the diagram (6 with broad $\mathrm{H} \alpha$ emission) and 101 galaxies in the composite region of the diagram (4 with broad $\mathrm{H} \alpha$ emission). An additional 15 galaxies have broad $\mathrm{H} \alpha$ emission, yet have HII-region-like narrow-line ratios. Middle: $[\mathrm{O} \mathrm{III}] / \mathrm{H} \beta$ versus $[\mathrm{S} \mathrm{II}] / \mathrm{H} \alpha$ diagram. Colors indicate classification based on the $\mathrm{OIII} / \mathrm{H} \beta$ versus $[\mathrm{N} \mathrm{II}] / \mathrm{H} \alpha$ diagram. Bottom: $[\mathrm{O}$ III $] / \mathrm{H} \beta$ versus $[\mathrm{O} \quad \mathrm{I}] / \mathrm{H} \alpha$ diagram for galaxies in which we detect the [O I] emission line (Tables 2 and (4). Colors indicate classification based on the $\mathrm{OIII} / \mathrm{H} \beta$ versus $[\mathrm{N} \mathrm{II}] / \mathrm{H} \alpha$ diagram. 


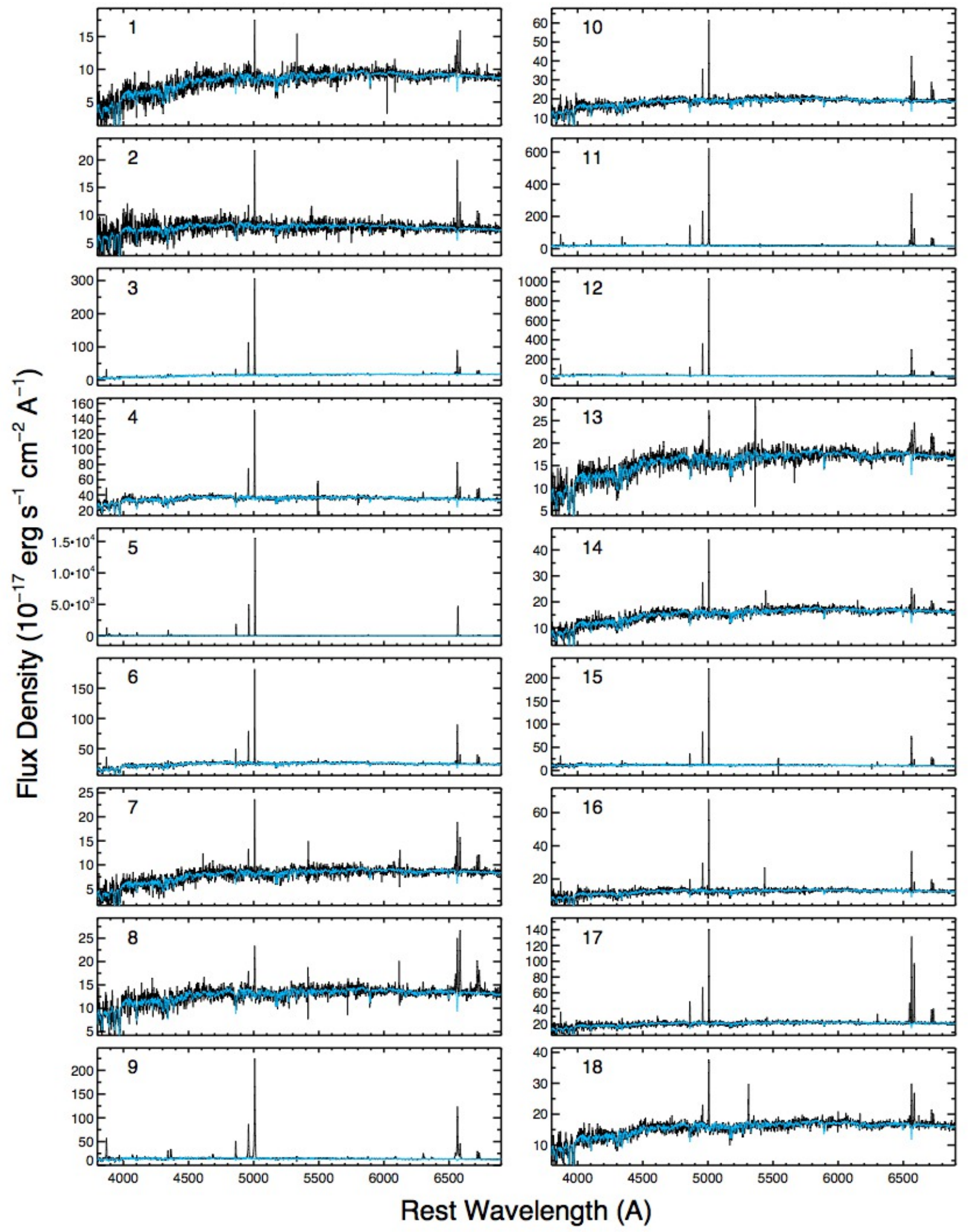

Figure 3. SDSS redshift-corrected spectra of galaxies falling in the AGN region of the $[\mathrm{O}$ III $] / \mathrm{H} \beta$ versus $[\mathrm{N}$ II $] / \mathrm{H} \alpha$ diagram. Continuum and absorption-line fits are shown in blue (see Section 3.1). An identification number (Table 1) is given in the upper left corner of each plot. 


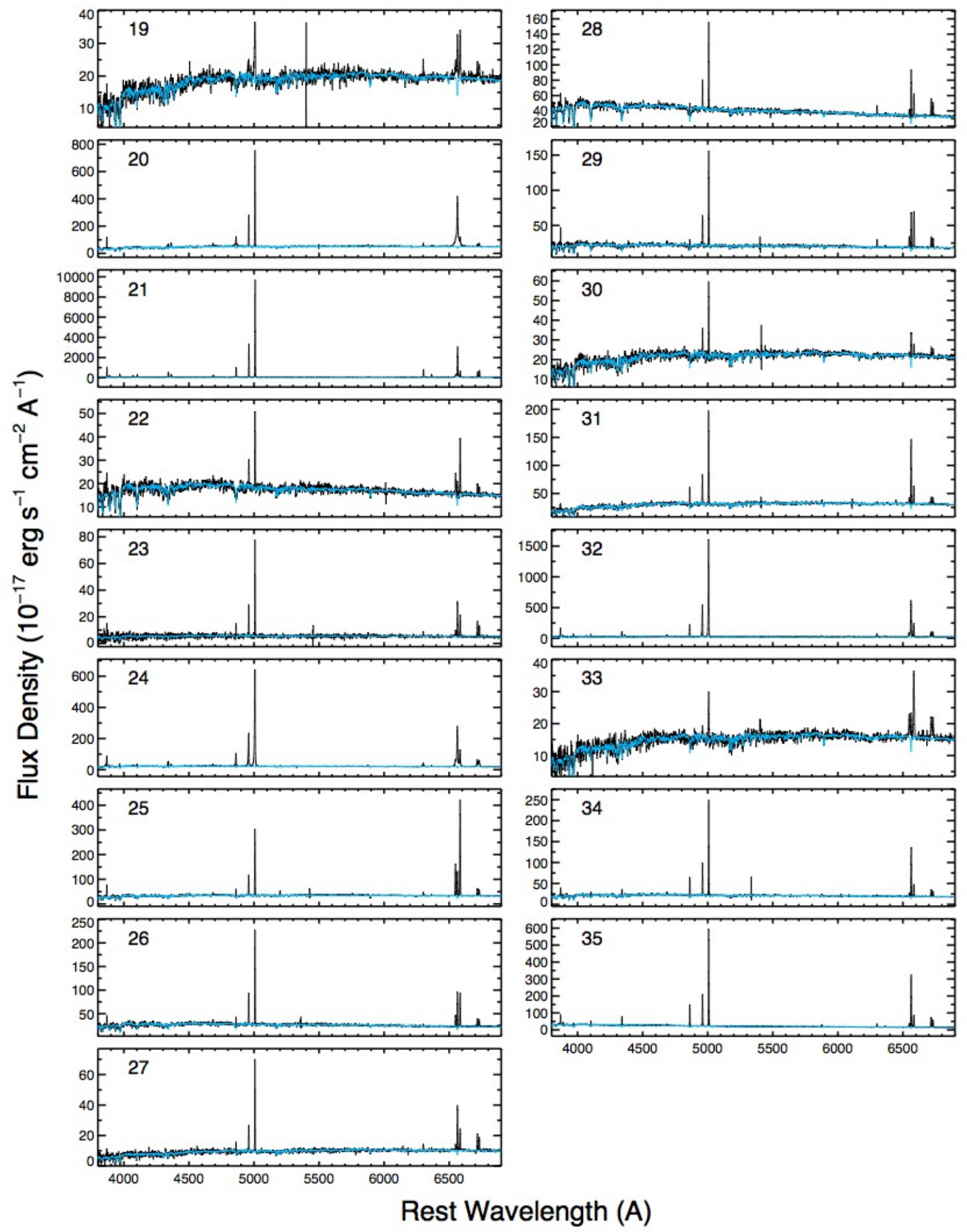

Figure 4. Same as Figure 3

parent dwarf catalog (2). The same is true of the four candidate metal-poor AGN presented in Izotov \& Thuan (2008). Our broad-line sample also includes HS $0837+4717$ (source B), which has narrow-line ratios consistent with a low-metallicity starburst or a low- metallicity AGN, and exhibits persistent broad emission lines (Kniazev et al. 2000, Izotov et al. 2007).

For each of our broad-line candidates, we check if the broad $\mathrm{H} \alpha$ component could be an artifact from oversubtracting the $\mathrm{H} \alpha$ absorption line. We fit a single Gaus- 


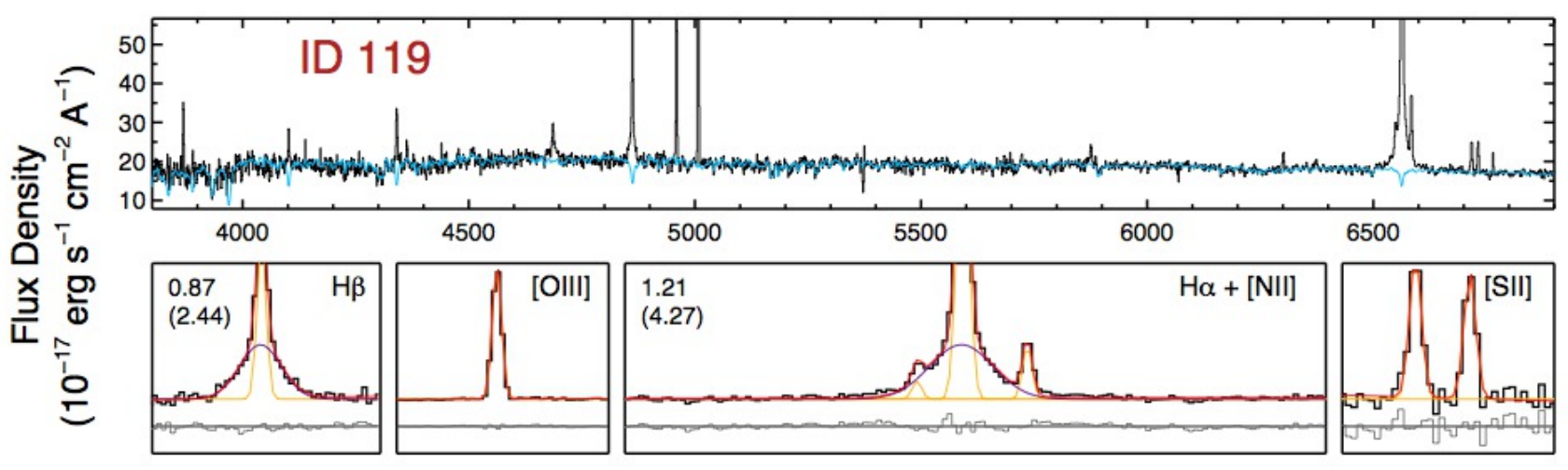

Rest Wavelength (A)

Figure 5. Example of a broad-line AGN candidate (ID 119). The narrow-line ratios of this source place it in the composite section of the $[\mathrm{O} \mathrm{III}] / \mathrm{H} \beta$ versus $[\mathrm{N} \mathrm{II}] / \mathrm{H} \alpha$ diagram. Top: The redshift-corrected spectrum with the continuum and absorption-line fit plotted in blue. Bottom: Chunks of the emission-line spectrum (after continuum and absorption-line subtraction). Best-fitting models are plotted in red and the individual narrow Gaussian components are plotted in yellow. The broad $\mathrm{H} \alpha$ and $\mathrm{H} \beta$ components are plotted in dark blue. Residuals are plotted in gray with a vertical offset for clarity. Reduced $\chi^{2}$ values are shown in the upper left-hand corner of the H $\alpha+$ $\left[\mathrm{N}\right.$ II] and $\mathrm{H} \beta$ chunks. For comparison, the reduced $\chi^{2}$ values from the fits not including a broad component are shown in parenthesis. Spectra of the other 24 broad-line AGN candidates are shown in the Appendix.

sian to the $\mathrm{H} \alpha$ absorption line in the model fit (Section 3.1), measure the corresponding equivalent width (EW) and FWHM, and compare these values to the broad $\mathrm{H} \alpha$ emission component. As shown in Figure 6, the EWs of the broad emission features are $\sim 3$ to 36 times the EWs of the absorption features and the FWHMs of the broad $\mathrm{H} \alpha$ components are $\sim 2$ to 11 times the FWHMs of the absorption lines. Moreover, in all but 1 case, the FWHMs of the $\mathrm{H} \alpha$ absorption lines are less than $500 \mathrm{~km}$ $\mathrm{s}^{-1}$, our minimum threshold for the width of any broad $\mathrm{H} \alpha$ emission. Thus, our broad $\mathrm{H} \alpha$ detections appear to be robust and not a result of over-subtracting an absorption feature.

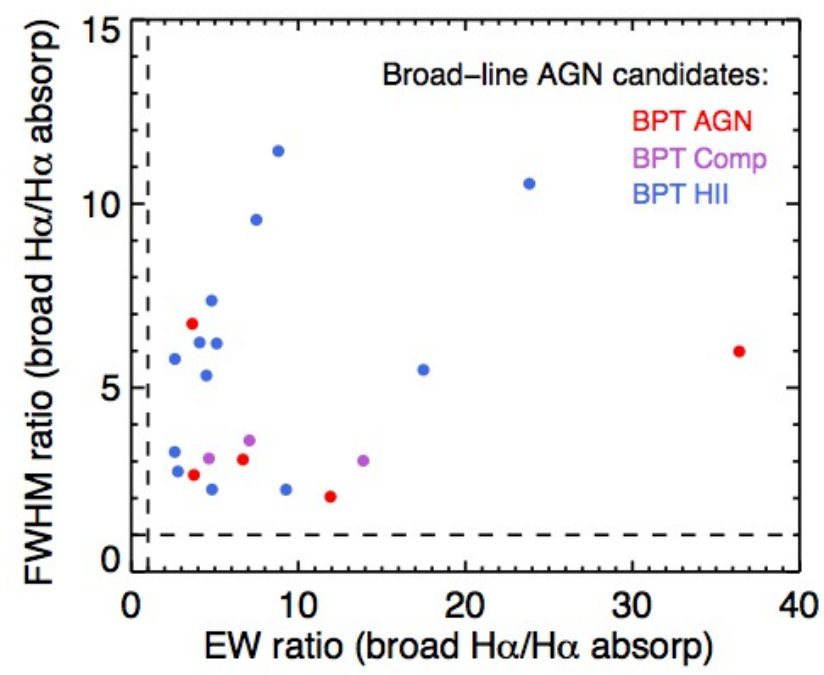

Figure 6. Ratios of the EWs and FWHMs of the broad components of $\mathrm{H} \alpha$ emission to the $\mathrm{H} \alpha$ absorption lines for the broad-line AGN candidates (Section 3.4). Points are color-coded according to their position in the $[\mathrm{O} \mathrm{III} / \mathrm{H} \beta$ versus $[\mathrm{N} \mathrm{II}] / \mathrm{H} \alpha$ BPT diagram. Dashed lines indicate ratios equal to 1 . This plot demonstrates that the broad $\mathrm{H} \alpha$ emission features are not a result of over-subtracting the $\mathrm{H} \alpha$ absorption features. Absorption lines were not detected in 4 of the 25 broad-line AGN candidates and they are excluded here.
The broad-line AGN candidates are found throughout the BPT diagrams (Figures 1 and 2), as is the case for other samples of Type 1 AGN (Greene \& Ho 2007a. Stern \& Laor 2013). Ten of the galaxies fall in either the AGN or composite region of the $[\mathrm{O}$ III $] / \mathrm{H} \beta$ vs. $[\mathrm{N} \mathrm{II}] / \mathrm{H} \alpha$ diagram and we consider these the most secure broadline AGN candidates, half of which are new identifications ${ }^{6}$ Accounting for just the BPT-AGN, the fraction of sources with a detectable broad $\mathrm{H} \alpha$ component (i.e., the Type 1 fraction) is $\sim 17 \%(6 / 35)$. The other 15 broad-line sources fall in the star-forming region of the $[\mathrm{O}$ III] $/ \mathrm{H} \beta$ vs. [N II] $/ \mathrm{H} \alpha$ diagram (Tables 3 and 4 ), 2 of which fall in the Seyfert (and 2 in the LINER) region of the $[\mathrm{O} \mathrm{III}] / \mathrm{H} \beta$ vs. $[\mathrm{O} \mathrm{I}] / \mathrm{H} \alpha$ diagram. While models of low-metallicity AGNs overlap with low-metallicity starbursts (Groves et al. 2006), only one of the broad-line sources in the star-forming part of the $[\mathrm{O}$ III] $/ \mathrm{H} \beta$ vs. $[\mathrm{N} \mathrm{II}] / \mathrm{H} \alpha$ diagram also has narrow-line ratios consistent with a low-metallicity AGN (source B). For the majority of cases, the narrow-lines are likely dominated by star formation. Bona fide broad-line AGN falling in the starforming part of the diagnostic diagram can naturally be explained by star-formation dominating the narrow-line emission within the $3^{\prime \prime}$ SDSS aperture, which can cover a substantial fraction of the host galaxy for these dwarfs. It is also possible, however, that the broad $\mathrm{H} \alpha$ seen in objects lying in the star-forming region of the BPT diagram is in fact from stellar phenomena.

Broad $\mathrm{H} \alpha$ from galaxies in the star-forming region of the BPT diagram may well be from luminous Type II SNe that happened to be detectable when the SDSS spectra were taken and fell within the spectroscopic aperture. Type II SNe can exhibit broad $\mathrm{H} \alpha$ emission with luminosities upwards of $\sim 10^{40} \mathrm{erg} \mathrm{s}^{-1}$, which is comparable to the luminosities of our broad-line sources and other examples of AGNs with low-mass BHs (Filippenko \& Ho 2003 Greene \& Ho 2007a). Some Type II SNe also exhibit broad $\mathrm{P}$ Cygni profiles in $\mathrm{H} \alpha$ and we have already excluded these sources from our broad-line sam-

\footnotetext{
6 Barth et al. (2008) identify a tentative broad component in ID 32 ('Table I).
} 
Table 3

BPT Star-Forming Galaxies with Broad H $\alpha$ : Galaxy Properties

\begin{tabular}{|c|c|c|c|c|c|c|c|c|c|}
\hline $\begin{array}{l}\text { ID } \\
(1)\end{array}$ & $\begin{array}{l}\text { NSAID } \\
(2)\end{array}$ & $\begin{array}{l}\text { SDSS Name } \\
(3)\end{array}$ & $\begin{array}{l}\text { Plate-MJD-Fiber } \\
(4)\end{array}$ & $\begin{array}{c}z \\
(5)\end{array}$ & $\underset{(6)}{\log M_{\star}}$ & $\begin{array}{l}M_{g} \\
(7)\end{array}$ & $\begin{array}{c}g-r \\
(8)\end{array}$ & $\begin{array}{l}r_{50} \\
(9)\end{array}$ & $\begin{array}{l}\text { Sérsic } n \\
\quad(10)\end{array}$ \\
\hline$A^{a}$ & 22083 & J004042.10-110957.7 & $655-52162-89$ & 0.0274 & 9.45 & -18.14 & 0.56 & 1.0 & 2.3 \\
\hline $\mathrm{B}^{\mathrm{b}}$ & 15952 & J084029.91+470710.4 & $549-51981-621$ & 0.0421 & 8.11 & -18.89 & -0.85 & 0.9 & 6.0 \\
\hline $\mathrm{C}$ & 109990 & J090019.66+171736.9 & $2432-54052-524$ & 0.0288 & 9.30 & -19.08 & 0.26 & 2.3 & 1.1 \\
\hline $\mathrm{D}$ & 76788 & J091122.24+615245.5 & $1786-54450-514$ & 0.0266 & 8.79 & -18.56 & 0.26 & 2.2 & 0.6 \\
\hline $\mathrm{E}$ & 109016 & $\mathrm{~J} 101440.21+192448.9$ & $2373-53768-148$ & 0.0289 & 8.75 & -17.90 & 0.23 & 0.9 & 1.9 \\
\hline $\mathrm{F}$ & 12793 & J105100.64+655940.7 & $490-51929-279$ & 0.0325 & 9.11 & -19.05 & 0.11 & 0.8 & 6.0 \\
\hline G & 13496 & J105447.88+025652.4 & $507-52353-619$ & 0.0222 & 8.90 & -18.56 & 0.20 & 0.9 & 2.9 \\
\hline $\mathrm{H}$ & 74914 & $\mathrm{~J} 111548.27+150017.7$ & $1752-53379-532$ & 0.0501 & 8.82 & -18.93 & 0.19 & 1.5 & 5.3 \\
\hline $\mathrm{I}$ & 112250 & $\mathrm{~J} 112315.75+240205.1$ & $2497-54154-221$ & 0.0250 & 9.01 & -18.33 & 0.44 & 0.8 & 6.0 \\
\hline $\mathrm{J}^{\mathrm{a}}$ & 41331 & $\mathrm{~J} 114343.77+550019.4$ & $1015-52734-596$ & 0.0272 & 9.01 & -17.97 & 0.22 & 1.1 & 0.9 \\
\hline $\mathrm{K}$ & 91579 & $\mathrm{~J} 120325.66+330846.1$ & $2089-53498-283$ & 0.0349 & 9.01 & -17.44 & 0.92 & 1.1 & 5.9 \\
\hline $\mathrm{L}$ & 33207 & $\mathrm{~J} 130724.64+523715.5$ & $887-52376-454$ & 0.0262 & 9.09 & -19.14 & 0.19 & 1.2 & 1.3 \\
\hline M & 119311 & $\mathrm{~J} 131503.77+223522.7$ & $2651-54507-488$ & 0.0230 & 9.14 & -18.90 & 0.29 & 1.7 & 3.9 \\
\hline $\mathrm{N}$ & 88972 & $\mathrm{~J} 131603.91+292254.0$ & 2009-53904-640 & 0.0378 & 8.93 & -19.72 & -0.14 & 1.4 & 3.1 \\
\hline $\mathrm{O}$ & 104565 & $\mathrm{~J} 134332.09+253157.7$ & $2246-53767-49$ & 0.0287 & 9.35 & -18.58 & 0.37 & 3.3 & 0.9 \\
\hline
\end{tabular}

Note. - Col.(1): Identification assigned in this paper. Col.(2): NSA identification number. Col.(3): SDSS name. Col.(4): Plate-MJDFiber of analyzed spectra. Col.(5): Redshift. Col.(6): Log galaxy stellar mass in units of $\mathrm{M}_{\odot}$. Col.(7): Absolute $g$-band magnitude. Col.(8): $g-r$ color. Col.(9): Petrosian 50\% light radius in units of kpc. Col.(10): Sérsic index, $n$. All values are from the NSA and assume $h=0.73$. Magnitudes are $K$-corrected to rest-frame values using kcorrect $v 4 \_2$ and corrected for foreground Galactic extinction.

a Galaxies in Greene \& Ho (2007) and Dong et al. (2012)

b HS $0837+4717$ [zotov et al. 2007). The $[\mathrm{O} \mathrm{III]} / \mathrm{H} \beta$ and $[\mathrm{N} \mathrm{II}] / \mathrm{H} \alpha$ ratios for this source are also consistent with a low-metallicity AGN

Table 4

BPT Star-Forming Galaxies with Broad $\mathrm{H} \alpha$ : Emission Line Fluxes

\begin{tabular}{|c|c|c|c|c|c|c|c|c|c|c|}
\hline $\begin{array}{l}\text { ID } \\
(1)\end{array}$ & $\begin{array}{c}(\mathrm{H} \beta)_{n} \\
(2)\end{array}$ & $\begin{array}{c}(\mathrm{H} \beta)_{b} \\
(3)\end{array}$ & $\begin{array}{c}{\left[\begin{array}{ll}\mathrm{O} & \mathrm{III}\end{array}\right] \lambda 5007} \\
(4)\end{array}$ & $\begin{array}{c}{\left[\begin{array}{ll}\mathrm{O} & \mathrm{I}] \\
(5) & \lambda 6300\end{array}\right.} \\
\end{array}$ & $\begin{array}{c}{[\mathrm{N}} \\
\mathrm{II}] \\
(6)\end{array}$ & $\begin{array}{c}(\mathrm{H} \alpha)_{n} \\
\quad(7)\end{array}$ & $\begin{array}{c}(\mathrm{H} \alpha)_{b} \\
(8)\end{array}$ & $\begin{array}{c}{[\mathrm{N} \text { II }] \lambda 6583} \\
(9)\end{array}$ & $\begin{array}{c}{[\mathrm{S}} \\
\mathrm{II}] \lambda 6716 \\
(10)\end{array}$ & $\begin{array}{c}{\left[\begin{array}{ll}\mathrm{S} & \mathrm{II}\end{array}\right] \lambda 6731} \\
(11)\end{array}$ \\
\hline A & $42(5)$ & & $52(6)$ & $21(5)$ & $9(1)$ & 113(6) & 218(17) & $26(3)$ & $44(4)$ & $32(3)$ \\
\hline B & $2287(47)$ & $195(20)$ & $14521(202)$ & $46(4)$ & $54(2)$ & $8066(82)$ & $1292(23)$ & $161(5)$ & $115(4)$ & $107(3)$ \\
\hline $\mathrm{C}$ & $340(6)$ & 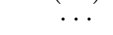 & $560(9)$ & $29(3)$ & $53(1)$ & $1155(14)$ & $749(21)$ & $157(3)$ & $190(4)$ & $141(3)$ \\
\hline D & $180(6)$ & $\ldots$ & $450(10)$ & $16(4)$ & $16(1)$ & $571(9)$ & $251(26)$ & $48(3)$ & $99(4)$ & $66(3)$ \\
\hline $\mathrm{E}$ & $408(10)$ & $\ldots$ & $1218(24)$ & $32(5)$ & $44(1)$ & $1348(20)$ & $107(17)$ & $129(4)$ & $170(6)$ & $125(4)$ \\
\hline $\mathrm{F}$ & $917(79)$ & $311(13)$ & $1241(39)$ & $38(5)$ & $361(31)$ & $3764(323)$ & $582(28)$ & $1069(77)$ & $293(57)$ & $263(89)$ \\
\hline G & 1058(19) & $\ldots$ & 1782(167) & $54(6)$ & $237(4)$ & $3567(45)$ & $365(27)$ & $702(12)$ & $518(11)$ & $373(7)$ \\
\hline $\mathrm{H}$ & $164(6)$ & $\ldots$ & 394(10) & $28(5)$ & $23(1)$ & $549(10)$ & $183(22)$ & $68(4)$ & $86(4)$ & $64(3)$ \\
\hline I & $576(14)$ & $\ldots$ & $664(15)$ & $62(7)$ & $215(4)$ & $2264(30)$ & $196(27)$ & $638(12)$ & $392(10)$ & $305(7)$ \\
\hline $\mathrm{J}$ & $227(9)$ & $80(18)$ & $299(10)$ & $17(5)$ & $39(2)$ & $1065(31)$ & $648(22)$ & $116(5)$ & $135(6)$ & $90(4)$ \\
\hline K & $183(5)$ & $\ldots$ & $330(6)$ & $30(3)$ & $39(1)$ & $655(9)$ & $155(10)$ & $114(3)$ & 133(4) & $95(2)$ \\
\hline $\mathrm{L}$ & $685(16)$ & $\ldots$ & $1405(26)$ & $63(7)$ & 111(3) & 2174(31) & $236(35)$ & $329(8)$ & $371(10)$ & $288(7)$ \\
\hline M & $495(11)$ & $\ldots$ & $441(20)$ & $15(5)$ & $154(4)$ & $1690(22)$ & $521(30)$ & $457(10)$ & $229(7)$ & $169(5)$ \\
\hline $\mathrm{N}$ & $3524(44)$ & $\ldots$ & $14615(162)$ & $302(16)$ & $365(4)$ & $11905(129)$ & $907(55)$ & $1080(13)$ & $981(15)$ & $727(10)$ \\
\hline $\mathrm{O}$ & $34(4)$ & $\ldots$ & $27(4)$ & $9(2)$ & 8(1) & $106(3)$ & $46(8)$ & $23(2)$ & $36(3)$ & $22(2)$ \\
\hline
\end{tabular}

Note. - Col.(1): Identification assigned in this paper. Col.(2)-(11): Emission line fluxes with units of $10^{-17} \mathrm{erg} \mathrm{s}^{-1} \mathrm{~cm}{ }^{-2}$. Errors are shown in parenthesis. No extinction correction has been applied. The subscripts $n$ and $b$ refer to the narrow and broad components of the line, respectively. A three-dot ellipsis indicates that no broad component of $\mathrm{H} \beta$ is detected.

ple. We identified 9 such objects by eye (Table 5), and these were subsequently confirmed by the automated SNe detection code used in Graur \& Maoz (2013) (O.Graur, private communication). Two objects, NSAID 119259 and NSAID 69982, have also been identified as Type II SNe by Izotov \& Thuan (2009a) and Izotov et al. (2007), respectively. Figure 7 shows the spectral region around $\mathrm{H} \alpha$ for the SNe candidates. In some cases, the P Cygni profile is very subtle with only slight blue-shifted absorption indicated by an asymmetric emission line profile. Another way we can identify SNe in our broad-line sample is to examine the temporal evolution of the broad $\mathrm{H} \alpha$ emission. We would expect the broad line to persist for an AGN, whereas it should significantly decrease or disappear over a timespan of several years for SNe. Therefore, we are currently obtaining follow-up spectroscopy of the broad-line sources, the results of which will be presented in a forthcoming paper.

We also consider stellar winds from evolving massive stars undergoing mass loss, such as those from WolfRayet (WR) stars and Luminous Blue Variables (LBVs). WR stars are identified in the integrated spectra of galaxies by the blended emission from helium, carbon and nitrogen at $\lambda 4650$ - 4690 known as the "WR bump" and this feature is only detected in four of our broad-line AGN candidates by Brinchmann et al. (2008)7. Two are BPT-AGN, including NGC 4395 and one of the galaxies from the Barth et al. (2008) sample (IDs 21 and 32 ), and two are BPT-star-forming galaxies (IDs B and $\mathrm{F})$. We note that broad He II $\lambda 4686$ can also be produced by AGN and thus the identifications of WR fea-

7 The work of Brinchmann et al. (2008) makes use of DR6, whereas we use DR8. 18 of our 25 broad-line AGN candidates are found in DR6. 
Table 5

Supernova Candidates

\begin{tabular}{cccccc}
\hline \hline $\begin{array}{c}\text { NSAID } \\
(1)\end{array}$ & $\begin{array}{c}\text { SDSS Name } \\
(2)\end{array}$ & $\begin{array}{c}\text { Plate-MJD-Fiber } \\
(3)\end{array}$ & $\begin{array}{c}z \\
(4)\end{array}$ & $\begin{array}{c}\log \mathrm{M}_{\star} \\
(5)\end{array}$ & $\begin{array}{c}M_{g} \\
(6)\end{array}$ \\
\hline 12271 & $\mathrm{~J} 093313.94+015858.7$ & $475-51965-626$ & 0.0311 & 8.94 & -17.81 \\
47648 & $\mathrm{~J} 082449.94+293644.1$ & $1207-52672-512$ & 0.0404 & 9.45 & -19.06 \\
61339 & $\mathrm{~J} 131307.11+460554.3$ & $1459-53117-22$ & 0.0296 & 9.46 & -18.40 \\
69671 & $\mathrm{~J} 162244.78+323933.0$ & $1684-53239-484$ & 0.0410 & 9.41 & -19.74 \\
$69982^{\mathrm{a}}$ & $\mathrm{J} 164402.63+273405.4$ & $1690-53475-360$ & 0.0232 & 8.93 & -17.80 \\
75038 & $\mathrm{~J} 113913.54+150215.7$ & $1755-53386-516$ & 0.0140 & 8.51 & -17.08 \\
117522 & $\mathrm{~J} 103134.64+190407.1$ & $2593-54175-334$ & 0.0342 & 8.89 & -17.92 \\
$119259^{\mathrm{b}}$ & $\mathrm{J} 132053.66+215510.2$ & $2651-54507-31$ & 0.0224 & 8.55 & -16.97 \\
160073 & $\mathrm{~J} 113322.89+550420.0$ & $1014-52707-463$ & 0.0091 & 8.65 & -18.16
\end{tabular}

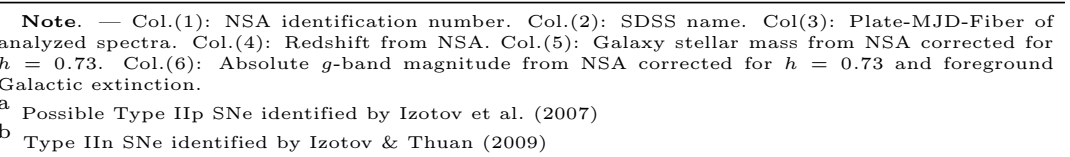

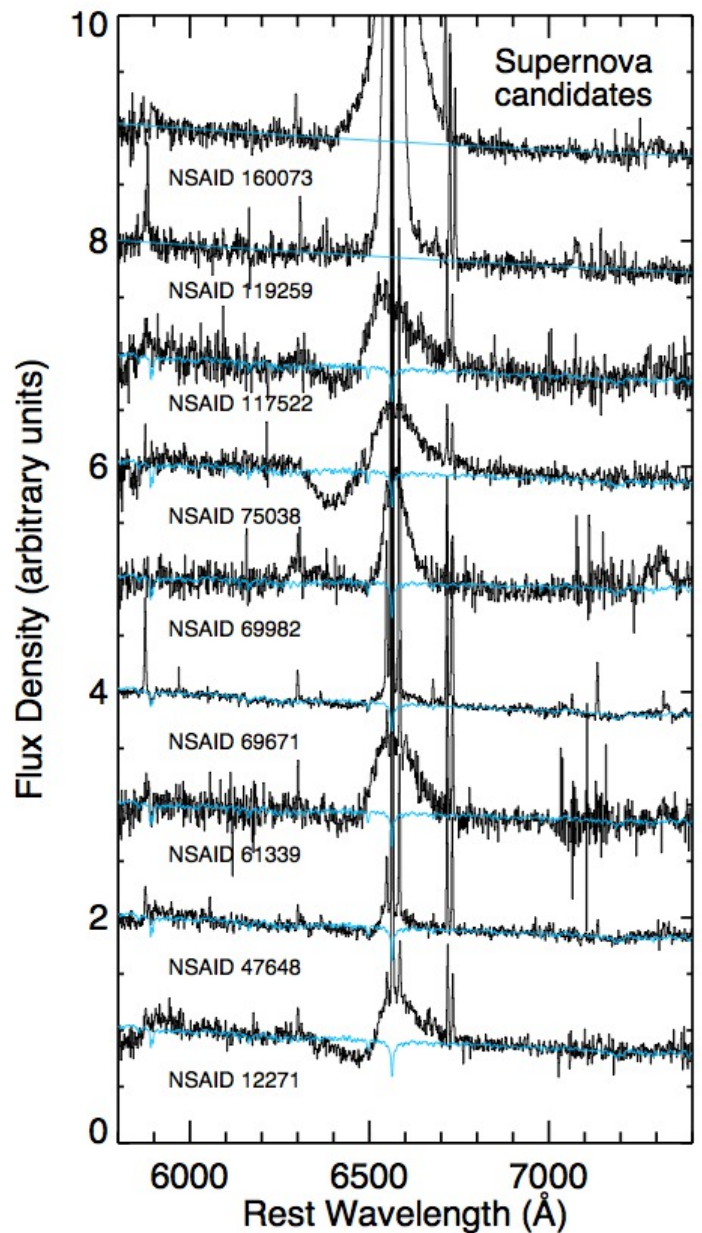

Figure 7. The 9 supernovae candidates excluded from our broadline AGN candidates. The spectral region around $\mathrm{H} \alpha$ is shown with the continuum and absorption line fits plotted in blue.

tures in these galaxies (especially the BPT-AGN) are suspect. Moreover, WR stars do not exhibit strong, if any, hydrogen lines (e.g. Schaerer \& Vacca 1998, Crowther 2007: Crowther \& Walborn 2011). Therefore, we do not consider WR stars a likely possibility for producing the observed broad $\mathrm{H} \alpha$ in the broad-line AGN candidates.
LBVs are sources of Balmer emission and can exhibit broad $\mathrm{H} \alpha$ with velocities as large as $\sim 1500 \mathrm{~km} \mathrm{~s}^{-1}$, yet they are generally less luminous than Type II SNe (Smith et al. 2011) and we therefore consider them a less likely source of possible contamination in the broad-line sample. Nevertheless, like SNe, LBVs are transient events that we can eliminate from our broad-line AGN candidates with our follow-up spectroscopic campaign.

In the spectra of some broad-line AGN candidates, the broad $\mathrm{H} \alpha$ components are rather offset from the narrow components. If a given broad $\mathrm{H} \alpha$ line is indeed from gas orbiting a massive BH, this may suggest the AGN is physically offset from the center of the galaxy (perhaps due to a relatively shallow potential well in a low-mass galaxy). Alternatively, the broad-line could be due to a SNe offset from the galaxy center, which is more likely for objects in the star-forming region of the BPT diagrams with exceptionally broad $\mathrm{Ha}$ (e.g, object C). Our followup observations will help discern the origin of the offset lines.

\subsection{Black Hole Masses}

For the objects with broad $\mathrm{H} \alpha$ emission, we can estimate indirect $\mathrm{BH}$ masses using scaling relations. If the BLR gas is virialized, for which there is some evidence (Peterson et al. 2004), then we can use the BLR kinematics as a dynamical tracer of the $\mathrm{BH}$ mass $\left(M_{\mathrm{BH}} \propto\right.$ $\left.R \Delta V^{2} / G\right)$. The average gas velocity is inferred from the emission-line width. The BLR size has been measured only for $\sim 50$ active galaxies using reverberation mapping (e.g., Peterson et al. 2004, Bentz et al. 2009b; Denney et al. 2010, Barth et al. 2011, Bentz et al. 2013), in which the time lag between continuum and BLR variability establishes a size scale for the BLR. In general, the geometry of the BLR (and the proportionality constant) is not known, and so the virial product alone $\left(R \Delta V^{2} / G\right)$ does not establish the absolute BH mass. Instead, the ensemble of reverberation-based $\mathrm{BH}$ masses are calibrated using the $M_{\mathrm{BH}}-\sigma_{\star}$ relation (Gebhardt et al. 2000b, Ferrarese et al.|2001; Nelson et al. 2004; Onken et al.||2004, Greene \& Ho 2006; Park et al. 2012, Grier et al. 2013), although for some caveats see Greene et al. (2010).

For all other broad-line AGNs, the BLR size is unknown, and is indirectly estimated using a correlation between AGN luminosity and BLR size (the radius- 
luminosity relation) that is measured using $\mathrm{H} \beta$ timelag measurements from the reverberation-mapped sample (e.g., Kaspi et al. 2005, Bentz et al. 2009a, 2013). Since the AGN continuum luminosity is virtually impossible to measure in these low-luminosity galaxies with ongoing star formation, we use the broad Balmer line luminosity as a proxy for the AGN luminosity (e.g., Yee 1980).

We follow the approach outlined in Greene \& Ho (2005b) to estimate virial BH masses using the FWHM and luminosity of broad $\mathrm{H} \alpha$, but with the modified radius-luminosity relationship of Bentz et al. (2013). Using the results from the "clean" fit in Bentz et al. (2013), the $R_{\mathrm{BLR}}-L$ relationship is given by

$$
\log \left(\frac{R_{\text {BLR }}}{l t-\text { days }}\right)=1.555+0.542 \log \left(\frac{L_{5100}}{10^{44} \mathrm{erg} \mathrm{s}^{-1}}\right) .
$$

Greene \& Ho (2005b) present well-defined empirical correlations between broad $\mathrm{H} \alpha$ luminosity and continuum luminosity, $L_{5100}$, and the line-widths (FWHM) of $\mathrm{H} \alpha$ and $\mathrm{H} \beta$ :

$$
\begin{gathered}
L_{\mathrm{H} \alpha}=5.25 \times 10^{42}\left(\frac{L_{5100}}{10^{44} \mathrm{erg} \mathrm{s}^{-1}}\right)^{1.157} \mathrm{erg} \mathrm{s}^{-1} \\
\mathrm{FWHM}_{\mathrm{H} \beta}=1.07 \times 10^{3}\left(\frac{\mathrm{FWHM}_{\mathrm{H} \alpha}}{10^{3} \mathrm{~km} \mathrm{~s}^{-1}}\right)^{1.03} \mathrm{~km} \mathrm{~s}^{-1}
\end{gathered}
$$

Inserting the previous three equations into the virial relationship:

$$
M_{\mathrm{BH}}=\epsilon\left(\frac{R_{\mathrm{BLR}} \mathrm{FWHM}_{\mathrm{H} \beta}^{2}}{G}\right),
$$

gives

$$
\begin{aligned}
\log \left(\frac{M_{\mathrm{BH}}}{M_{\odot}}\right)=\log \epsilon+6.57 & \left.+0.47 \log \left(\frac{L_{\mathrm{H}_{\alpha}}}{10^{42} \mathrm{erg} \mathrm{s}^{-1}}\right) 5\right) \\
& +2.06 \log \left(\frac{\mathrm{FWHM}_{\mathrm{H} \alpha}}{10^{3} \mathrm{~km} \mathrm{~s}^{-1}}\right) .
\end{aligned}
$$

Several values of the scale factor, $\epsilon$, are found in the literature 8 , spanning a range of $\sim 0.75-1.4$ (e.g., Onken et al. 2004 Greene \& Ho 2007a; Grier et al. 2013). Here we assume $\epsilon=1$.

Table 6 lists the broad $\mathrm{H} \alpha$ luminosities, widths, and virial $\mathrm{BH}$ masses for our sample of broad-line AGN candidates. The most secure broad-line AGN candidates (i.e. those that lie in the AGN or composite region of the $\mathrm{BPT}$ diagram) have broad $\mathrm{H} \alpha$ luminosities of $\sim 10^{39}-10^{40}$ erg $\mathrm{s}^{-1}$, with the exception of the nearby dwarf Seyfert NGC 4395 that has a broad $\mathrm{H} \alpha$ luminosity of $\sim 10^{38}$ $\operatorname{erg~} \mathrm{s}^{-1}$. For these sources, the widths (FWHM) of the broad $\mathrm{H} \alpha$ components span a range of $\sim 600-1600$ $\mathrm{km} \mathrm{s}^{-1}$. The virial $\mathrm{BH}$ masses calculated using Equation 6 are in the range $\sim 10^{5}-10^{6}$ with a median of

\begin{tabular}{|c|c|c|c|}
\hline $\begin{array}{l}\text { ID } \\
(1)\end{array}$ & $\begin{array}{c}\log L(\mathrm{H} \alpha)_{b} \\
(2)\end{array}$ & $\underset{(3)}{\mathrm{FWHM}}(\mathrm{H} \alpha)_{b}$ & $\underset{(4)}{\log M_{\mathrm{BH}}}$ \\
\hline \multicolumn{4}{|c|}{$B P T A G N s$} \\
\hline 1 & 39.38 & 1577 & 5.7 \\
\hline 9 & 40.15 & 703 & 5.4 \\
\hline 11 & 39.41 & 636 & 4.9 \\
\hline 20 & 40.13 & 1526 & 6.1 \\
\hline 21 & 38.15 & 1288 & 5.0 \\
\hline 32 & 39.73 & 747 & 5.2 \\
\hline \multicolumn{4}{|c|}{ BPT Composites } \\
\hline 48 & 39.67 & 894 & 5.4 \\
\hline 119 & 40.16 & 1043 & 5.7 \\
\hline 123 & 39.82 & 634 & 5.1 \\
\hline 127 & 39.45 & 792 & 5.2 \\
\hline \multicolumn{4}{|c|}{ BPT Star-Forming } \\
\hline $\mathrm{A}$ & 39.52 & 1782 & 5.9 \\
\hline $\mathrm{B}$ & 40.67 & 1245 & 6.1 \\
\hline $\mathrm{C}$ & 40.10 & 3690 & 6.8 \\
\hline $\mathrm{D}$ & 39.56 & 4124 & 6.7 \\
\hline $\mathrm{E}$ & 39.26 & 935 & 5.2 \\
\hline $\mathrm{F}$ & 40.09 & 598 & 5.2 \\
\hline $\mathrm{G}$ & 39.56 & 2027 & 6.1 \\
\hline $\mathrm{H}$ & 39.97 & 3014 & 6.6 \\
\hline I & 39.39 & 994 & 5.3 \\
\hline $\mathrm{J}$ & 39.99 & 1521 & 6.0 \\
\hline $\mathrm{K}$ & 39.58 & 774 & 5.2 \\
\hline $\mathrm{L}$ & 39.51 & 3126 & 6.4 \\
\hline M & 39.75 & 3563 & 6.6 \\
\hline $\mathrm{N}$ & 40.42 & 645 & 5.4 \\
\hline $\mathrm{O}$ & 38.88 & 1563 & 5.5 \\
\hline
\end{tabular}

\footnotetext{
8 We follow Onken et al. (2004) and denote the scale factor as $\epsilon$ when using the FWHM of the line, as opposed to $f$ when using the second moment of the line profile, $\sigma$.
}

Table 6

Broad-line AGN Candidates: BH Masses

\footnotetext{
Note. - Col.(1): Identification assigned in this paper. Col.(2): Luminosity of the broad component of $\mathrm{H} \alpha$ in units of erg s${ }^{-1}$. Col.(3): FWHM of the broad component of $\mathrm{H} \alpha$, corrected for instrumental resolution, in units of $\mathrm{km}$ $\mathrm{s}^{-1}$. Col.(4): Virial mass estimate of the $\mathrm{BH}$ in units of $\mathrm{M}_{\odot}$, assuming the broad $\mathrm{H} \alpha$ emission is due to accretion. $\mathrm{As}$ described in the text, the origin of the broad emission for the BPT star-forming galaxies is unclear at present.
}

$\sim 2 \times 10^{5} \mathrm{M}_{\odot}$. For comparison, the median BH masses in the Greene \& Ho (2007a) and Dong et al. (2012) samples are $\sim 1 \times 10^{6} \mathrm{M}_{\odot}$. The less secure broad-line sources (in the star-forming region of the BPT diagram) have broad $\mathrm{H} \alpha$ luminosities comparable to the more secure broadline AGN candidates. However, the widths reach values of a few thousand $\mathrm{km} \mathrm{s}^{-1}$, leading to anomalously large $\mathrm{BH}$ mass estimates and possibly indicating a different origin for the broad $\mathrm{H} \alpha$ emission at least in the most extreme cases (Figure 8a, also see Section 3.4).

The virial BH mass measurements for the broad-line AGN candidates are obviously extremely indirect, and are subject to a number of systematic uncertainties. For one thing, the BLR geometry clearly varies considerably from object to object (Kollatschny 2003. Bentz et al. 2009b; Denney et al. 2010, Barth et al. 2011), making a single geometric scaling factor suspect. Whether the geometry depends on fundamental parameters of the $\mathrm{BH}$ remains uncertain (Collin et al. 2006). Then there is the danger that we are not measuring the velocities at the same radius as probed with reverberation mapping (Krolik 2001). At present, there simply are not enough measurements to search for such systematics directly, although progress with velocity-resolved reverberation mapping is promising (Gaskell 1988, Kollatschny 2003. Bentz et al. 2008; Denney et al. 2009 Barth et al. 2011). Finally, reverberation mapping has been achieved in only a few low-mass AGNs (Peterson et al. 2005, Rafter et al. 2011) and we therefore strongly caution that the $\mathrm{BH}$ 
masses presented here are based on an extrapolation from more luminous AGNs powered by more massive BHs and living in very different environments.

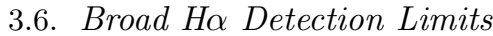

For the BPT AGN and composites that do not have detectable broad $\mathrm{H} \alpha$ emission in their spectra, we determine upper limits on any potential broad $\mathrm{H} \alpha$ flux. We add fake broad $\mathrm{H} \alpha$ components represented by Gaussians to the emission-line spectra and measure the emission lines with the code described in Section 3.2. The input FWHM is held fixed at $500 \mathrm{~km} \mathrm{~s}^{-1}$ to match our selection criteria and we incrementally increase the height of the Gaussian until our code flags the source as having a broad $\mathrm{H} \alpha$ component. The minimum detectable flux is $\sim 10^{-15} \mathrm{erg} \mathrm{s}^{-1} \mathrm{~cm}^{-2}$ within a factor of $\sim 3$ for nearly all of the sources. This corresponds to a broad $\mathrm{H} \alpha$ luminosity of $\sim 2 \times 10^{39} \mathrm{erg} \mathrm{s}^{-1}$ at a median redshift of $z \sim 0.03$. We note that all of the BPT AGN and composites with detected broad $\mathrm{H} \alpha$ are above this threshhold, with the exception of NGC 4395 that is only $\sim 4$ Mpc away.

We estimate the bolometric luminosity of an AGN corresponding to our minimum detectable broad $\mathrm{H} \alpha$ luminosity using the conversion between $L(\mathrm{H} \alpha)$ and $L(5100 \AA)$ in Equation 2 (Greene \& Ho 2005b) and $L_{\text {bol }}=10.3 L(5100 \AA)$ Richards et al. 2006). We caution that these relationships come from studies of more luminous Seyfert 1 galaxies and quasars. Nevertheless, we use these relationships and estimate that an AGN must have a minimum bolometric luminosity of $L_{\mathrm{bol}} \gtrsim 10^{42} \mathrm{erg} \mathrm{s}^{-1}$ for us to detect broad $\mathrm{H} \alpha$ emission (modulo obscuration from dust).

In principle, we could detect broad $\mathrm{H} \alpha$ from a $\mathrm{BH}$ with a mass as low as $\sim 8 \times 10^{3} \mathrm{M}_{\odot}$ if it was accreting at its Eddington limit, where $L_{\mathrm{Edd}}=1.3 \times 10^{38}\left(M_{\mathrm{BH}} / \mathrm{M}_{\odot}\right)$ $\mathrm{erg} \mathrm{s} \mathrm{s}^{-1}$. Since maximally accreting $\mathrm{BHs}$ are very rare (Schulze \& Wisotzki 2010), it is not surprising that we do not detect such low-mass BHs (if they exist) in the limited volume of our sample $(z<0.055)$. In other words, we cannot rule out the existence of $\sim 10^{4} \mathrm{M}_{\odot}$ BHs. We just cannot detect them via broad $\mathrm{H} \alpha$ if they are radiating much below their Eddington luminosity. A $10^{5} \mathrm{M}_{\odot}$ $\mathrm{BH}$, on the other hand, only needs to be radiating at $\sim 8 \%$ of its Eddington luminosity to produce detectable broad $\mathrm{H} \alpha$ within our survey volume.

\section{HOST GALAXIES}

The NSA, from which we have drawn our parent sample of dwarfs (Section 2), provides a number of galaxy parameters. In Figure $8 \mathrm{p}-\mathrm{f}$, we plot the distributions of various properties for the 136 galaxies that exhibit ionization signatures of $\mathrm{BH}$ accretion in their spectra (i.e., those galaxies with narrow-line ratios falling in the AGN and composite region of the BPT diagram). We do not include the 15 broad-line AGN candidates falling in the star-forming region of the BPT diagram for the reasons discussed in Section 3.4. For comparison, we also show the distributions for our parent sample of emission-line galaxies normalized to the same number sources (136), as well as the 35 BPT-AGNs alone.

By design, the galaxies in our sample have stellar masses $M_{\star} \lesssim 3 \times 10^{9} \mathrm{M}_{\odot}$, which is approximately the stellar mass of the LMC (van der Marel et al. 2002).
While active BHs are preferentially found in more massive galaxies within our parent sample, we do detect active BHs in galaxies 10 times less massive than our threshold with stellar masses comparable to the Small Magellanic Cloud (SMC, $M_{\star} \sim 3 \times 10^{8} \mathrm{M}_{\odot}$; Stanimirović et al. 2004). Stellar masses in the NSA are derived from kcorrect (Blanton \& Roweis 2007) using Galaxy Evolution Explorer (GALEX) UV and SDSS ugriz bands combined, and while they do not account for any possible AGN contribution, we do not expect the active BHs in our sample to significantly impact stellar mass estimates of the host galaxies. The use of many broadband filters should help mitigate the contribution from strong emission lines, and the vast majority of sources are type 2 AGN that are known to contribute very little continuum emission (e.g., Schmitt et al. 1999). For the small fraction of type 1 AGN candidates, the host galaxy stellar masses could in principle be artificially elevated by a boost in flux from the AGN continuum. However, HST I-band imaging of two of our broad-line AGN candidates (IDs 20 and 123; Jiang et al. 2011) that are also in the sample of low-mass BHs presented by Greene \& Ho (2007a) reveals that the AGNs in these galaxies contribute $\lesssim 10 \%$ of the total flux.

The active galaxies in our sample have a total (host $+\mathrm{AGN})$ median absolute $g$-band magnitude of $\left\langle M_{g}\right\rangle=$ -18.1 mag, which is comparable to the LMC $\left(M_{g}^{\mathrm{LMC}} \sim\right.$ $-18.2 \mathrm{mag}$; Tollerud et al. 2011) and $\sim 1-2$ magnitudes fainter than previous samples of low-mass AGN. Correcting the median absolute magnitudes to our adopted cosmology $(h=0.73)$, the Barth et al. (2008) sample of lowmass Seyfert 2 galaxies has $\left\langle M_{a}\right\rangle=-19.0$, and the lowmass Seyfert 1 samples of Greene \& Ho (2007a) and Dong et al. (2012) have $\left\langle M_{g}\right\rangle=-19.4 \mathrm{mag}$ and $\left\langle M_{g}\right\rangle=-20.3$ mag, respectively, after removing the AGN contribution.

The colors of our sample of dwarfs hosting active BHs tend to be redder compared to our parent sample of dwarfs. The distribution of colors remains essentially the same for the parent sample even when applying a mass cut of $\log M_{\star}>9.25$, and therefore the color difference between the active sample and parent sample is not a mass effect. The $A_{V}$ 's of the active galaxies derived from our continuum and absorption line fits are similar to the parent sample $(\sim 0$ to 1 with a median of $\sim 0.3)$, suggesting the redder colors are not due to differences in dust properties. More likely, the color difference is a selection effect such that the optical diagnostics we are using are not sensitive to accreting $\mathrm{BHs}$ in blue galaxies with ongoing star-formation that dominates the emission-line spectra.

In addition to being low-mass, the active galaxies in our sample are physically small, with typical half-light radii $r_{50} \lesssim 2 \mathrm{kpc}$. The distribution of BPT-AGN and composites is skewed towards smaller sizes relative to our parent sample of dwarf galaxies. A similar trend is seen in the distribution of $90 \%$-light radii, suggesting the galaxies hosting active $\mathrm{BH}$ s are indeed preferentially compact and this result is not due to the presence of a bright central point source.

Single-component two-dimensional Sérsic fits are also provided in the NSA and the galaxies in our sample span a large range of Sérsic index, $n$. Relative to our parent sample, a larger fraction of the active galaxies have high- 

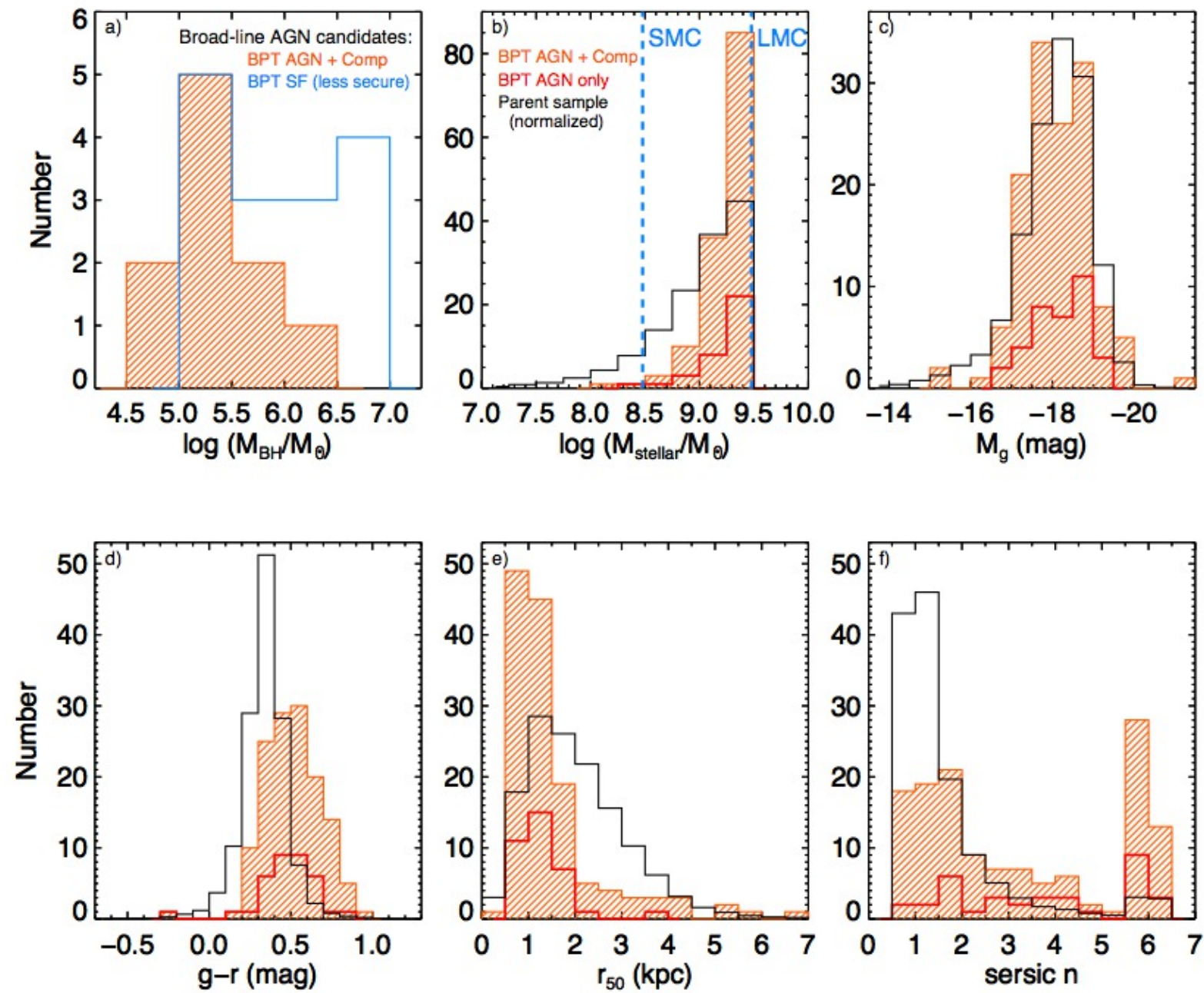

Figure 8. Panel a): Virial BH mass distribution for broad-line AGN candidates. BPT AGNs and composites are shown in orange. BPT star-forming galaxies, for which the origin of the broad-line emission is somewhat ambiguous, are shown in blue. Panels $b-f)$ : Distributions of host galaxy properties provided in the NSA for the 136 galaxies with narrow-line ionization signatures of BH accretion. BPT AGNs and composites are shown in the orange hashed histograms, and the distributions of BPT-AGNs only are shown in red. Our parent sample of dwarf emission-line galaxies is shown in black, normalized to the number of galaxies in the orange histogram (136 BPT AGN + composites). We show the distributions of galaxy stellar masses (with stellar masses of the Magellanic Clouds indicated in blue), total absolute $g$-band magnitude, $g-r$ color, Petrosian 50\% light radius, and Sérsic index. All magnitudes are $K$-corrected to rest-frame values using kcorrect v4_2 and corrected for foreground Galactic extinction. NSA values have been modified assuming $h=0.73$.

$n$ values, although $\mathrm{BHs}$ are also found in low- $n$ disky galaxies. Figure 9 shows the SDSS images of a selection of galaxies in our sample with $\mathrm{BH}$ accretion signatures.

\section{DEMOGRAPHICS}

The fraction of optically-selected, active $\mathrm{BHs}$ in our parent sample of dwarf galaxies is $\sim 0.5 \%(136 / 25974)^{9}$ However, there are a number of obstacles preventing us from determining the true occupation fraction and $\mathrm{BH}$ mass function in this low-mass regime. First of all, our optical diagnostics are only sensitive to actively accreting BHs, and even at their Eddington limit low-mass BHs are relatively faint. Furthermore, small galaxies generally have ongoing star formation, gas, and dust that can mask or extinguish the optical signatures of $\mathrm{BH}$ accretion. Therefore, while there may an accreting $\mathrm{BH}$ present

\footnotetext{
9 Including the additional 15 broad-line AGN candidates in the star-forming region of the BPT diagram does not have a significant impact on the active fraction, increasing it to $\sim 0.6 \%$.
}

at the center of a galaxy, the total observed line emission in the SDSS aperture may be dominated by star formation. Indeed, the SDSS aperture of $3^{\prime \prime}$ is comparable to the median half-light radius of our dwarf galaxy sample. Even without significant ongoing star formation, AGN signatures may be heavily diluted by host galaxy light such that the emission lines are effectively hidden (Moran et al. 2002). Additionally, low-metallicty AGN, which may be expected in lower-mass galaxies, can fall (and hide) in the upper left region of the star-forming plume of galaxies in the $[\mathrm{O} \mathrm{III}] / \mathrm{H} \beta$ versus $[\mathrm{N} \mathrm{II}] / \mathrm{H} \alpha$ diagnostic diagram (Groves et al. 2006). Therefore, while we can identify bona-fide AGNs based on their location in the BPT diagram, the selection of massive BHs is likely highly incomplete. Even if we understand our incompleteness from these effects, to derive a true space density requires that we know the distribution of Eddington ratios in these low-mass systems as compared to more massive galaxies (e.g., Heckman et al. 2004; Gallo et al. 

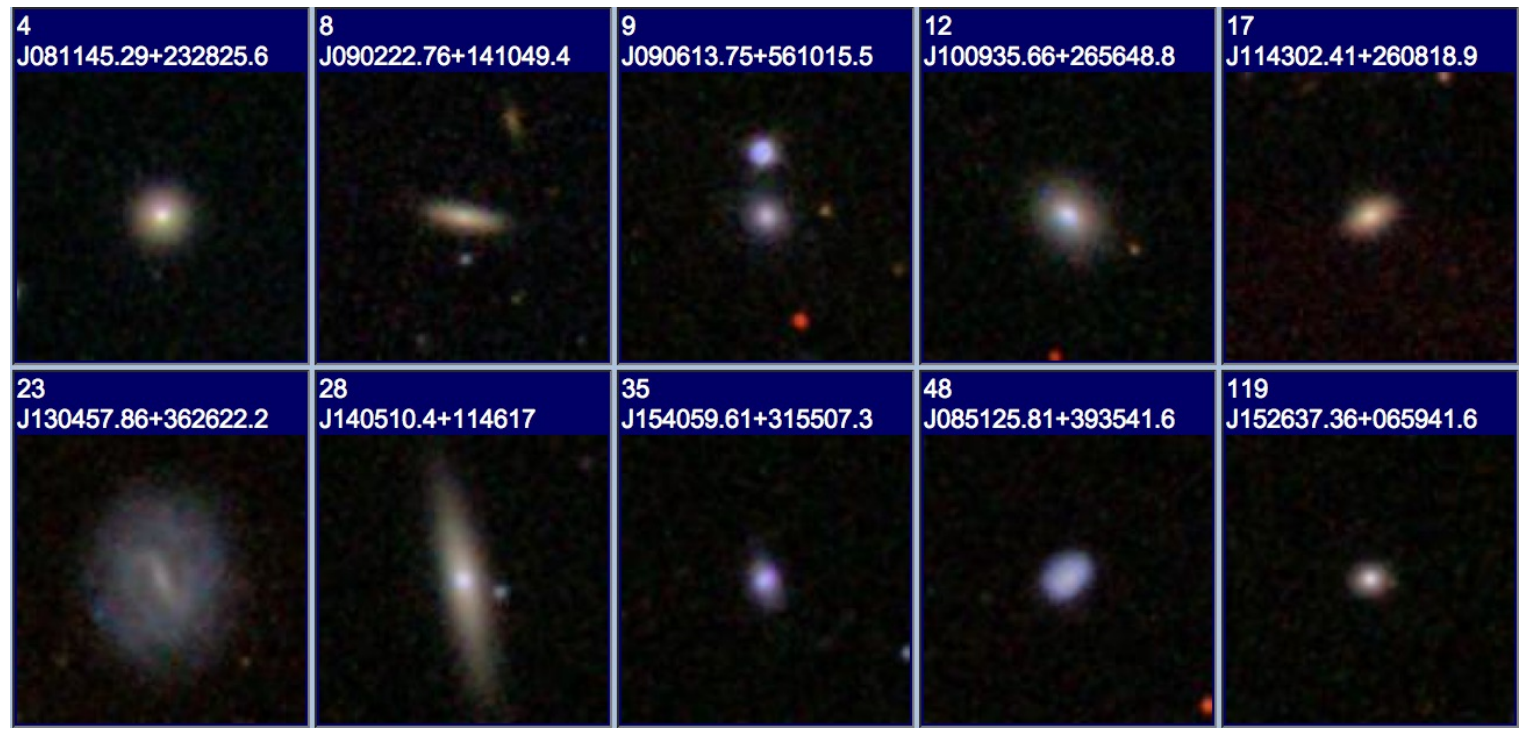

Figure 9. A selection of galaxies from our sample of dwarfs hosting active massive BHs. The SDSS color composite images have a size of $50^{\prime \prime} \times 50^{\prime \prime}$. The identification numbers assigned in this work are in the upper left corners of the images, with the SDSS name below.

2010, Aird et al. 2012) where we believe the occupation fraction is close to unity. It is interesting to note, however, that our active fraction is quite similar to that of $\sim 10^{7} \mathrm{M}_{\odot}$ BHs radiating at $\sim 10 \%$ of their Eddington limit (Heckman et al. 2004, Greene \& Ho 2007b).

Using broad emission lines to identify AGN in dwarf galaxies poses a different set of problems. The broadline signature is weaker for low-mass BHs and can be difficult to detect in galaxy-dominated spectra. There is also the possibility that the broad-line region disappears altogether below some critical luminosity or Eddington ratio (e.g., Nicastro 2000, Laor 2003; Elitzur \& Ho 2009, Trump et al. 2011; Marinucci et al. 2012). There are a number of candidate "true" Type 2 AGNs (e.g., Tran 2003 Bianchi et al. 2008) that show no sign of a broadline region in direct or polarized light, and no clear signs of obscuration in X-rays. It is thus possible that the Type 1 fraction drops towards lower luminosity, which could add significant complications in attempting to use these AGN as a tracer of the demographics of $\mathrm{BHs}$ in dwarf galaxies.

\section{CONCLUSIONS}

Using optical spectroscopy from the SDSS, we have systematically assembled the largest sample of dwarf galaxies $\left(10^{8.5} \lesssim M_{\star} \lesssim 10^{9.5} \mathrm{M}_{\odot}\right)$ hosting massive $\mathrm{BHs}$ to date. These dwarf galaxies have stellar masses comparable to the Magellanic Clouds and contain some of the least-massive supermassive BHs known. Contrary to common lore, low-mass, physically small dwarf galaxies can indeed form massive BHs.

We find photoionization signatures of $\mathrm{BH}$ accretion in 136 galaxies using the narrow-line $\left[\begin{array}{ll}\mathrm{O} & \mathrm{III}\end{array}\right] / \mathrm{H} \beta$ versus $\left[\begin{array}{ll}\mathrm{N} & \mathrm{II}\end{array}\right] / \mathrm{H} \alpha$ diagram as our primary diagnostic. Of these, 35 have AGN-dominated spectra and 101 have composite spectra suggesting ionization from both an AGN and massive stars. For the small fraction of these active galaxies with detectable broad $\mathrm{H} \alpha$ emission, we estimate a median virial $\mathrm{BH}$ mass of $M_{\mathrm{BH}} \sim 2 \times 10^{5} \mathrm{M}_{\odot}$.
Our sensitivity to broad $\mathrm{H} \alpha$ emission limits our ability to detect broad-line AGN with $\mathrm{BH}$ masses much below $\sim 10^{5} \mathrm{M}_{\odot}$ radiating at less than $\sim 10 \%$ of their Eddington luminosity. We find broad $\mathrm{H} \alpha$ in an additional 15 galaxies, yet their spectra exhibit narrow-line ratios consistent with star-forming galaxies. We caution that at these low-luminosities and low-metallicities, particularly for galaxies with high star formation rates, we are susceptible to contamination from stellar processes.

Ultimately, we need a complete census of massive BHs in dwarf galaxies to place stringent constraints on theories for the formation of supermassive $\mathrm{BH}$ seeds. While optical diagnostics certainly have a role to play, we need to move towards using alternative search techniques and observations at other wavelengths to make further progress (e.g., radio and X-ray; Reines et al.|2011, Reines \& Deller 2012, Gallo et al.2010; Miller et al. 2012, Kamizasa et al. 2012).

We are grateful to the entire SDSS collaboration for providing the data that made this work possible, to Michael Blanton and all those involved in creating the NASA-Sloan Atlas, and to Craig Markwardt for making his MPFIT code publicly available. We thank the referee for a very helpful review that improved the paper. A.E.R. appreciates helpful discussions with Mark Whittle, Jong-Hak Woo and Marta Volonteri. Support for A.E.R. was provided by NASA through the Einstein Fellowship Program, grant PF1-120086. J.E.G. is partially supported by an Alfred P. Sloan fellowship. 
APPENDIX

SPECTRA OF THE BROAD-LINE AGN CANDIDATES

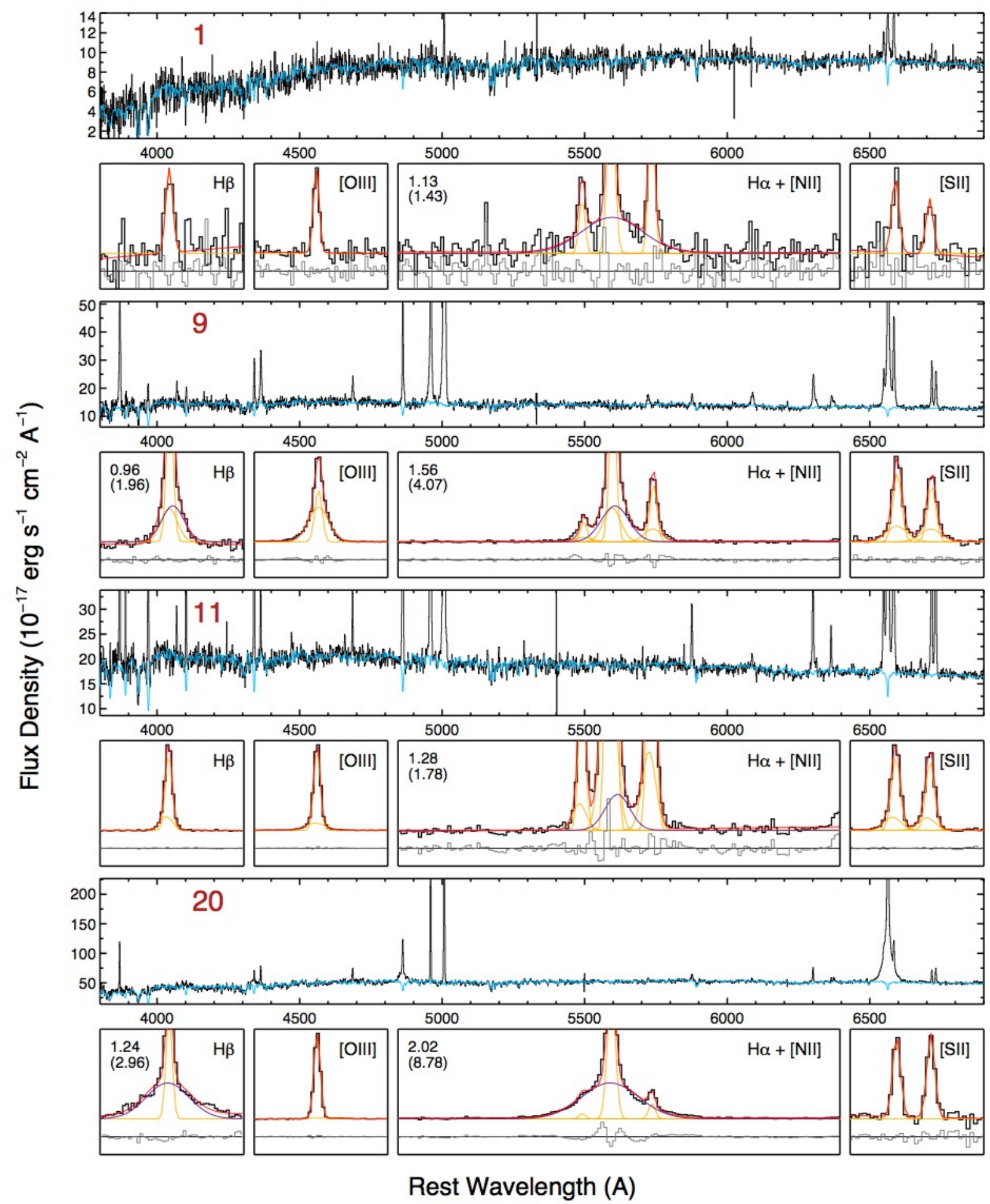

Figure 10. Same as Figure 5 


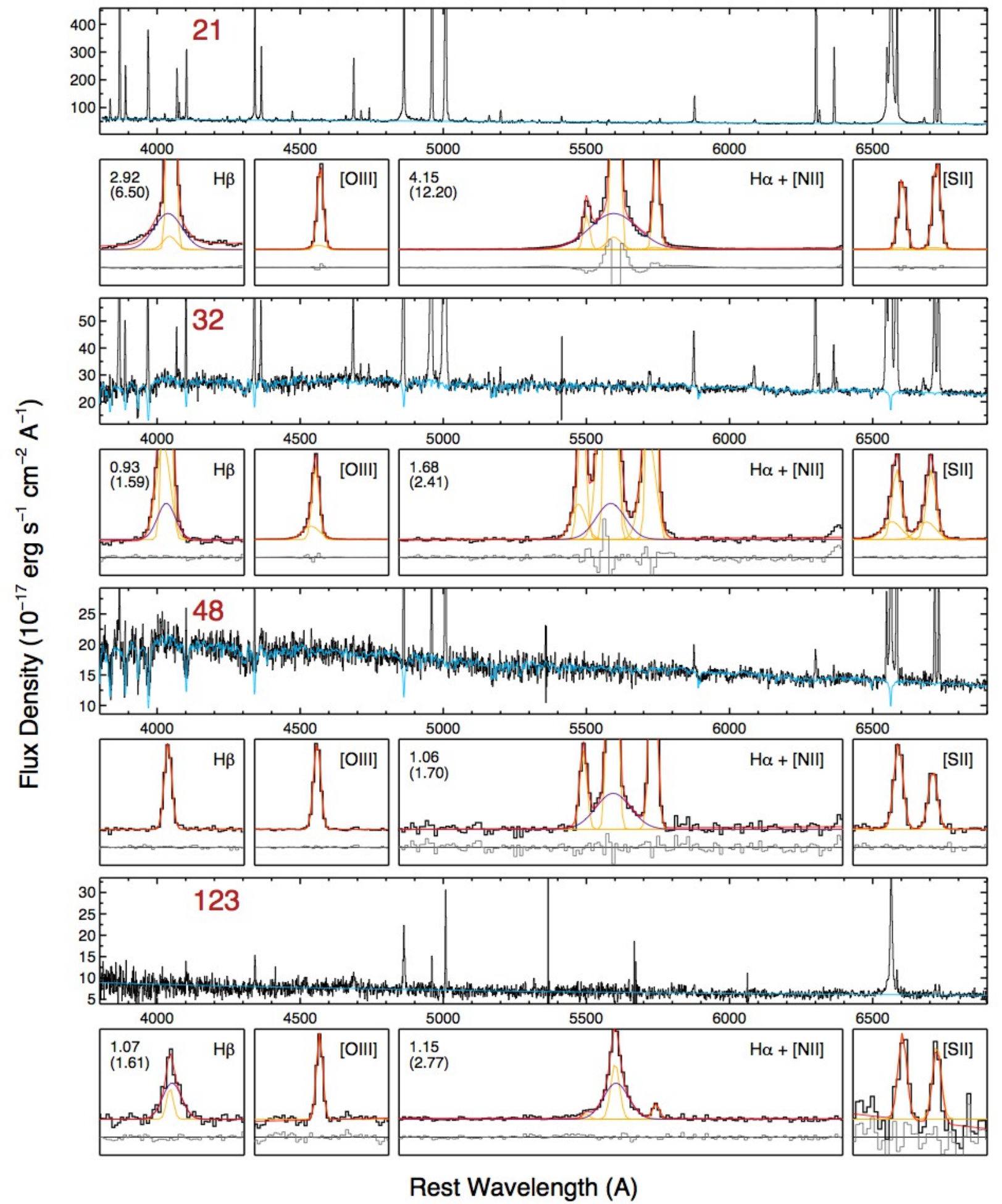

Figure 11. Same as Figure 5 Source 119 is shown in Figure 5 


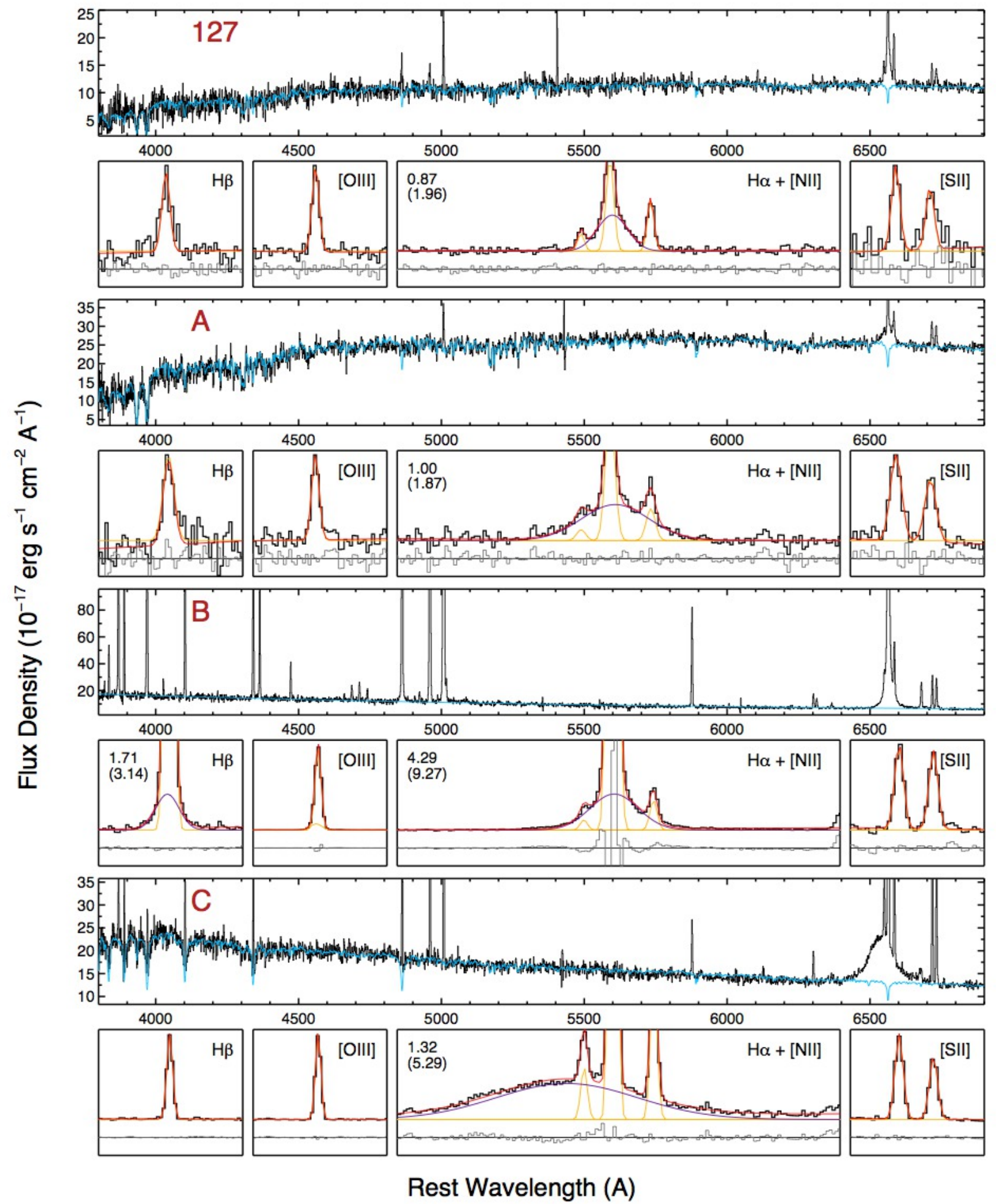

Figure 12. Same as Figure 5 


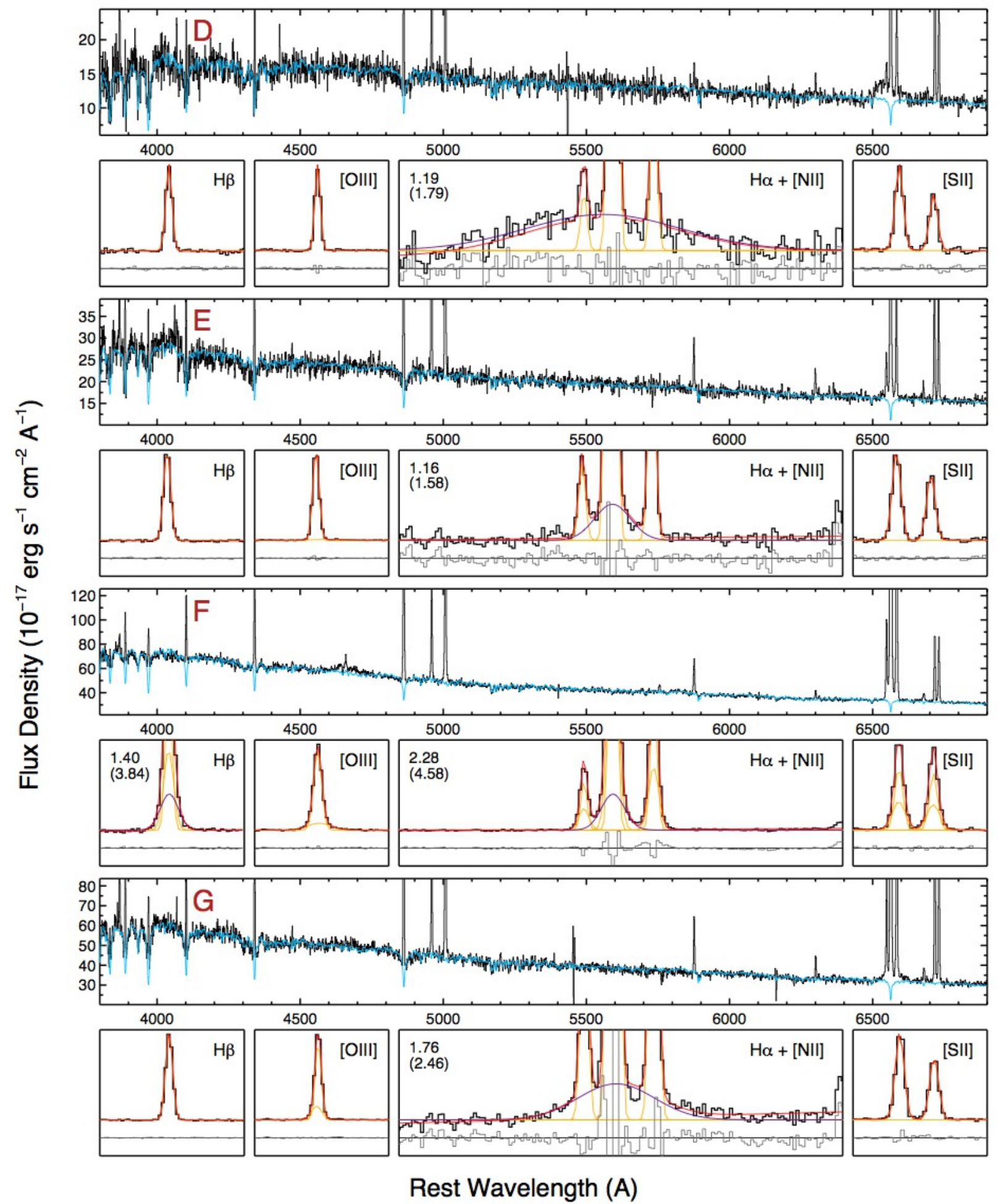

Figure 13. Same as Figure 5 


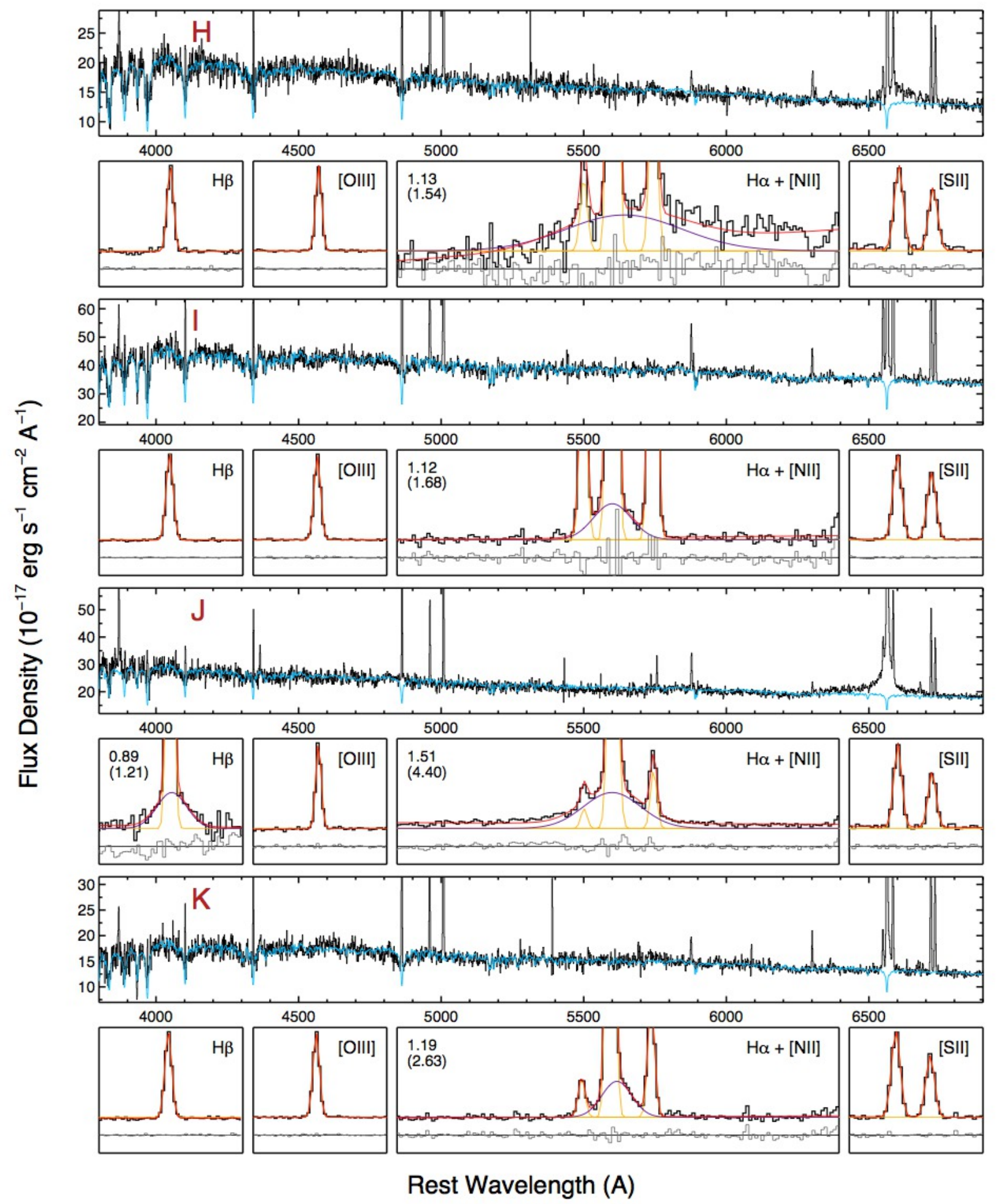

Figure 14. Same as Figure 5 


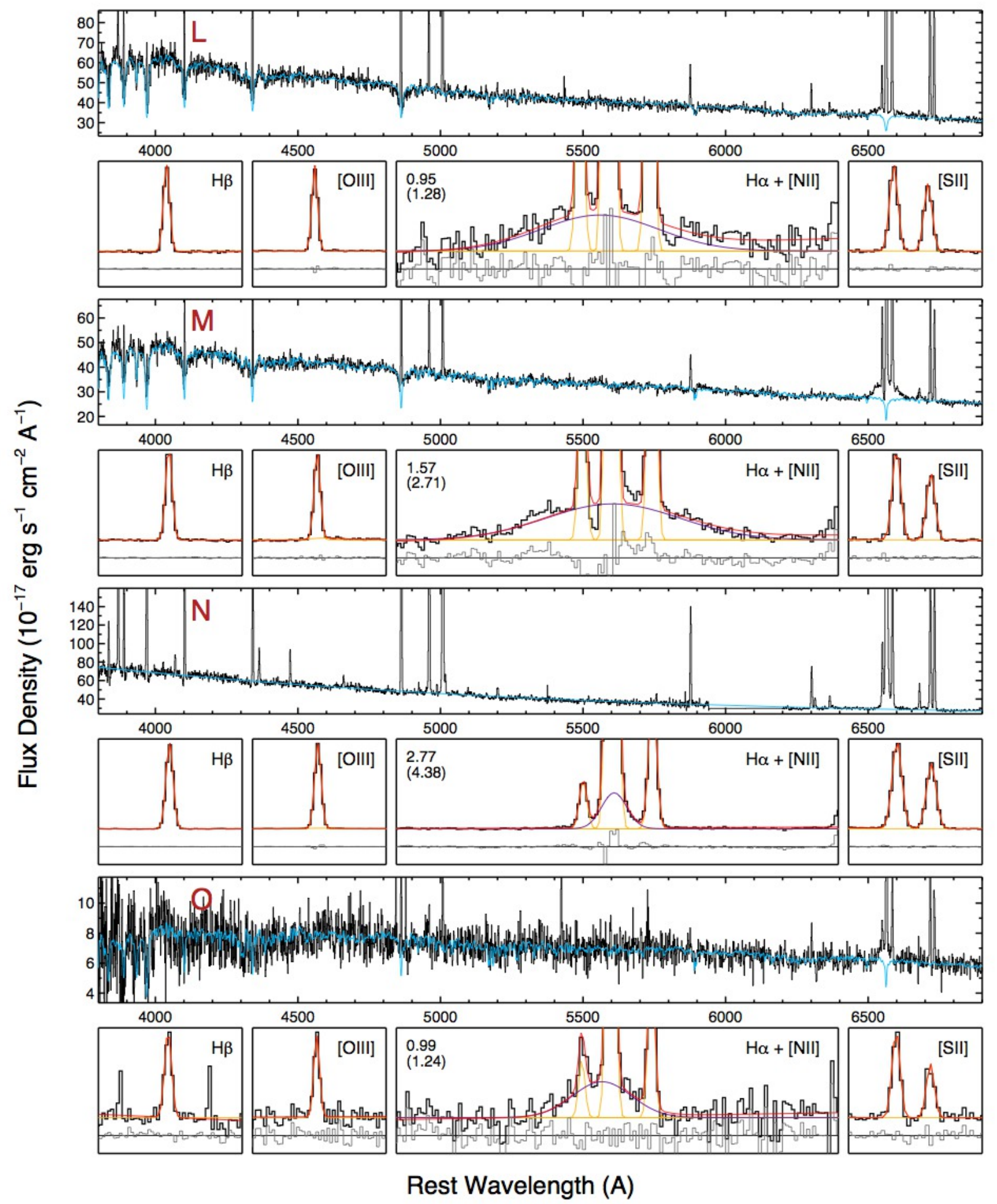

Figure 15. Same as Figure 5 


\section{REFERENCES}

Aihara, H., Allende Prieto, C., An, D., et al. 2011, ApJS, 193, 29

Aird, J., Coil, A. L., Moustakas, J., et al. 2012, ApJ, 746, 90

Alexandroff, R., et al. 2012, MNRAS, 423, 1325

Anders, P., \& Fritze-v. Alvensleben, U. 2003, A\&A, 401, 1063

Baldwin, J. A., Phillips, M. M., \& Terlevich, R. 1981, PASP, 93, 5

Barth, A. J., Greene, J. E., \& Ho, L. C. 2008, AJ, 136, 1179

Barth, A. J., Ho, L. C., Rutledge, R. E., \& Sargent, W. L. W. 2004, ApJ, 607, 90

Barth, A. J., Strigari, L. E., Bentz, M. C., Greene, J. E., \& Ho, L. C. 2009, ApJ, 690, 1031

Barth, A. J., Pancoast, A., Thorman, S. J., et al. 2011, ApJ, 743, L4

Begelman, M. C., Volonteri, M., \& Rees, M. J. 2006, MNRAS, 370, 289

Bell, E. F., McIntosh, D. H., Katz, N., \& Weinberg, M. D. 2003, ApJS, 149, 289

Bellovary, J., Volonteri, M., Governato, F., et al. 2011, ApJ, 742, 13

Bentz, M. C., Peterson, B. M., Netzer, H., Pogge, R. W., \& Vestergaard, M. 2009a, ApJ, 697, 160

Bentz, M. C., et al. 2008, ApJ, 689, L21

-. 2009b, ApJ, 705, 199

Bentz, M. C., Denney, K. D., Grier, C. J., et al. 2013, ApJ, 767, 149

Bianchi, S., Corral, A., Panessa, F., et al. 2008, MNRAS, 385, 195

Blanton, M. R., Kazin, E., Muna, D., Weaver, B. A., \& Price-Whelan, A. 2011, AJ, 142, 31

Blanton, M. R., \& Roweis, S. 2007, AJ, 133, 734

Brinchmann, J., Kunth, D., \& Durret, F. 2008, A\&A, 485, 657

Bromm, V., \& Yoshida, N. 2011, ARA\&A, 49, 373

Bruzual, G., \& Charlot, S. 2003, MNRAS, 344, 1000

Cardelli, J. A., Clayton, G. C., \& Mathis, J. S. 1989, ApJ, 345, 245

Collin, S., Kawaguchi, T., Peterson, B. M., \& Vestergaard, M. 2006, A\&A, 456, 75

Croton, D. J., Springel, V., White, S. D. M., et al. 2006, MNRAS, 365, 11

Crowther, P. A. 2007, ARA\&A, 45, 177

Crowther, P. A., \& Walborn, N. R. 2011, MNRAS, 416, 1311

Denney, K. D., Peterson, B. M., Pogge, R. W., et al. 2009, ApJ, 704, L80

- . 2010, ApJ, 721, 715

Desroches, L.-B., \& Ho, L. C. 2009, ApJ, 690, 267

Dong, X., Wang, T., Yuan, W., et al. 2007, ApJ, 657, 700

Dong, X.-B., Ho, L. C., Yuan, W., et al. 2012, ApJ, 755, 167

Dressler, A., \& Richstone, D. O. 1988, ApJ, 324, 701

Elitzur, M., \& Ho, L. C. 2009, ApJ, 701, L91

Eracleous, M., Hwang, J. A., \& Flohic, H. M. L. G. 2010, ApJ, 711, 796

Fan, X., et al. 2001, AJ, 122, 2833

Ferrarese, L., \& Merritt, D. 2000, ApJ, 539, L9

Ferrarese, L., Pogge, R. W., Peterson, B. M., et al. 2001, ApJ, 555, L79

Filippenko, A. V., \& Ho, L. C. 2003, ApJ, 588, L13

Filippenko, A. V., \& Sargent, W. L. W. 1988, ApJ, 324, 134

—. 1989, ApJ, 342, L11

Gallo, E., Treu, T., Jacob, J., et al. 2008, ApJ, 680, 154

Gallo, E., Treu, T., Marshall, P. J., et al. 2010, ApJ, 714, 25

Gaskell, C. M. 1988, ApJ, 325, 114

Gebhardt, K., et al. 2000a, ApJ, 539, L13

-. 2000b, ApJ, 543, L5

-. 2001, AJ, 122, 2469

Geha, M., Blanton, M. R., Yan, R., \& Tinker, J. L. 2012, ApJ, 757, 85

Ghosh, H., Mathur, S., Fiore, F., \& Ferrarese, L. 2008, ApJ, 687, 216

Goulding, A. D., Alexander, D. M., Lehmer, B. D., \& Mullaney, J. R. 2010, MNRAS, 406, 597

Graur, O., \& Maoz, D. 2013, MNRAS, 430, 1746

Greene, J. E. 2012, Nature Communications, 3, arXiv:1211.7082

Greene, J. E., \& Ho, L. C. 2004, ApJ, 610, 722

—. 2005a, ApJ, 627, 721

-. 2005b, ApJ, 630, 122

-. 2006, ApJ, 641, L21

-. 2007a, ApJ, 670, 92

-. 2007b, ApJ, 667, 131

Greene, J. E., Ho, L. C., \& Barth, A. J. 2008, ApJ, 688, 159

Greene, J. E., et al. 2010, ApJ, 721, 26

Grier, C. J., Martini, P., Watson, L. C., et al. 2013, ArXiv e-prints, arXiv:1305.2447

Groves, B. A., Heckman, T. M., \& Kauffmann, G. 2006, MNRAS, 371, 1559

Gültekin, K., Richstone, D. O., Gebhardt, K., et al. 2009, ApJ, 698, 198

Gunn, J. E., Siegmund, W. A., Mannery, E. J., et al. 2006, AJ, 131, 2332

Haehnelt, M. G., \& Rees, M. J. 1993, MNRAS, 263, 168

Hao, L., Strauss, M. A., Tremonti, C. A., et al. 2005, AJ, 129, 1783

Heckman, T. M. 1980, A\&A, 87, 152

Heckman, T. M., Kauffmann, G., Brinchmann, J., et al. 2004, ApJ, 613, 109

Heckman, T. M., Miley, G. K., van Breugel, W. J. M., \& Butcher, H. R. 1981, ApJ, 247, 403

Ho, L. C. 2008 , ARA\&A, 46, 475

Ho, L. C., Filippenko, A. V., Sargent, W. L. W., \& Peng, C. Y. 1997, ApJS, 112, 391

Hughes, S. A. 2002, MNRAS, 331, 805

Izotov, Y. I., \& Thuan, T. X. 2008, ApJ, 687, 133

-. 2009a, ApJ, 707, 1560 
-. 2009b, ApJ, 690, 1797

Izotov, Y. I., Thuan, T. X., \& Guseva, N. G. 2007, ApJ, 671, 1297

Jardel, J. R., \& Gebhardt, K. 2012, ApJ, 746, 89

Jia, J., Ptak, A., Heckman, T. M., et al. 2011, ApJ, 731, 55

Jiang, Y.-F., Greene, J. E., Ho, L. C., Xiao, T., \& Barth, A. J. 2011, ApJ, 742, 68

Kamizasa, N., Terashima, Y., \& Awaki, H. 2012, ApJ, 751, 39

Kaspi, S., Maoz, D., Netzer, H., et al. 2005, ApJ, 629, 61

Kauffmann, G., et al. 2003a, MNRAS, 341, 54

-. 2003b, MNRAS, 346, 1055

Kewley, L. J., Dopita, M. A., Sutherland, R. S., Heisler, C. A., \& Trevena, J. 2001, ApJ, 556, 121

Kewley, L. J., Groves, B., Kauffmann, G., \& Heckman, T. 2006, MNRAS, 372, 961

Kimm, T., Somerville, R. S., Yi, S. K., et al. 2009, MNRAS, 394, 1131

Kniazev, A. Y., Pustilnik, S. A., Ugryumov, A. V., \& Kniazeva, T. F. 2000, Astronomy Letters, 26, 129

Kollatschny, W. 2003, A\&A, 407, 461

Kormendy, J. 2004, in in Coevolution of Black Holes and Galaxies (Cambridge: Cambridge University Press), ed. L. C. Ho, 169

Kormendy, J., \& Ho, L. C. 2013, ArXiv e-prints, arXiv:1304.7762

Kormendy, J., \& Richstone, D. 1995, ARA\&A, 33, 581

Krolik, J. H. 2001, ApJ, 551, 72

Kunth, D., Sargent, W. L. W., \& Bothun, G. D. 1987, AJ, 93, 29

Laor, A. 2003, ApJ, 590, 86

Liu, X., Shapley, A. E., Coil, A. L., Brinchmann, J., \& Ma, C.-P. 2008, ApJ, 678, 758

Lodato, G., \& Natarajan, P. 2006, MNRAS, 371, 1813

Lora, V., Sánchez-Salcedo, F. J., Raga, A. C., \& Esquivel, A. 2009, ApJ, 699, L113

Ludwig, R. R., Greene, J. E., Barth, A. J., \& Ho, L. C. 2012, ApJ, 756, 51

Marconi, A., \& Hunt, L. K. 2003, ApJ, 589, L21

Marinucci, A., Bianchi, S., Nicastro, F., Matt, G., \& Goulding, A. D. 2012, ApJ, 748, 130

Markwardt, C. B. 2009, in Astronomical Society of the Pacific Conference Series, Vol. 411, Astronomical Data Analysis Software and Systems XVIII, ed. D. A. Bohlender, D. Durand, \& P. Dowler, 251

Mateo, M. L. 1998, ARA\&A, 36, 435

McConnachie, A. W. 2012, AJ, 144, 4

McConnell, N. J., \& Ma, C.-P. 2013, ApJ, 764, 184

Merloni, A., Heinz, S., \& di Matteo, T. 2003, MNRAS, 345, 1057

Miller, B., Gallo, E., Treu, T., \& Woo, J.-H. 2012, ApJ, 747, 57

Miller, J. M., \& Gültekin, K. 2011, ApJ, 738, L13

Miller, M. C., \& Davies, M. B. 2012, ApJ, 755, 81

Milosavljević, M., Bromm, V., Couch, S. M., \& Oh, S. P. 2009, ApJ, 698, 766

Moran, E. C., Eracleous, M., Leighly, K. M., et al. 2005, AJ, 129, 2108

Moran, E. C., Filippenko, A. V., \& Chornock, R. 2002, ApJ, 579, L71

Mortlock, D. J., Warren, S. J., Venemans, B. P., et al. 2011, Nature, 474, 616

Moustakas, J., Kennicutt, Jr., R. C., \& Tremonti, C. A. 2006, ApJ, 642, 775

Nelson, C. H., Green, R. F., Bower, G., Gebhardt, K., \& Weistrop, D. 2004, ApJ, 615, 652

Neumayer, N., \& Walcher, C. J. 2012, Advances in Astronomy, 2012, arXiv:1201.4950

Nicastro, F. 2000, ApJ, 530, L65

Onken, C. A., Ferrarese, L., Merritt, D., et al. 2004, ApJ, 615, 645

Panessa, F., Wolter, A., Pellegrini, S., et al. 2005, ApJ, 631, 707

Park, D., Woo, J.-H., Treu, T., et al. 2012, ApJ, 747, 30

Peterson, B. M., et al. 2004, ApJ, 613, 682

-. 2005, ApJ, 632, 799

Portegies Zwart, S. F., Baumgardt, H., Hut, P., Makino, J., \& McMillan, S. L. W. 2004, Nature, 428, 724

Rafter, S. E., Kaspi, S., Behar, E., Kollatschny, W., \& Zetzl, M. 2011, ApJ, 741, 66

Reines, A. E., \& Deller, A. T. 2012, ApJ, 750, L24

Reines, A. E., Nidever, D. L., Whelan, D. G., \& Johnson, K. E. 2010, ApJ, 708, 26

Reines, A. E., Sivakoff, G. R., Johnson, K. E., \& Brogan, C. L. 2011, Nature, 470, 66

Richards, G. T., et al. 2006, ApJS, 166, 470

Rosswog, S., Ramirez-Ruiz, E., \& Hix, W. R. 2009, ApJ, 695, 404

Sarzi, M., et al. 2006, MNRAS, 366, 1151

Satyapal, S., Böker, T., Mcalpine, W., et al. 2009, ApJ, 704, 439

Satyapal, S., Vega, D., Dudik, R. P., Abel, N. P., \& Heckman, T. 2008, ApJ, 677, 926

Satyapal, S., Vega, D., Heckman, T., O'Halloran, B., \& Dudik, R. 2007, ApJ, 663, L9

Schaerer, D., \& Vacca, W. D. 1998, ApJ, 497, 618

Schmitt, H. R., Storchi-Bergmann, T., \& Fernandes, R. C. 1999, MNRAS, 303, 173

Schramm, M., Silverman, J. D., Greene, J. E., et al. 2013, ArXiv e-prints, arXiv:1305.3826

Schulze, A., \& Wisotzki, L. 2010, A\&A, accepted (astroph/1004.2671), arXiv:1004.2671

Seth, A. C., et al. 2010, ApJ, 714, 713

Shen, Y., Greene, J. E., Strauss, M. A., Richards, G. T., \& Schneider, D. P. 2008, ApJ, 680, 169

Smith, N., Li, W., Silverman, J. M., Ganeshalingam, M., \& Filippenko, A. V. 2011, MNRAS, 415, 773

Stanimirović, S., Staveley-Smith, L., \& Jones, P. A. 2004, ApJ, 604, 176

Stern, J., \& Laor, A. 2013, MNRAS, 431, 836

Strubbe, L. E., \& Quataert, E. 2009, MNRAS, 400, 2070

Thornton, C. E., Barth, A. J., Ho, L. C., Rutledge, R. E., \& Greene, J. E. 2008, ApJ, 686, 892

Tollerud, E. J., Boylan-Kolchin, M., Barton, E. J., Bullock, J. S., \& Trinh, C. Q. 2011, ApJ, 738, 102

Tran, H. D. 2003, ApJ, 583, 632

Tremonti, C. A., Heckman, T. M., Kauffmann, G., et al. 2004, ApJ, 613, 898

Trouille, L., Barger, A. J., \& Tremonti, C. 2011, ApJ, 742, 46

Trump, J. R., Impey, C. D., Kelly, B. C., et al. 2011, ApJ, 733, 60

Valluri, M., Ferrarese, L., Merritt, D., \& Joseph, C. L. 2005, ApJ, 628, 137

van der Marel, R. P., Alves, D. R., Hardy, E., \& Suntzeff, N. B. 2002, AJ, 124, 2639 
van der Marel, R. P., Cretton, N., de Zeeuw, P. T., \& Rix, H.-W. 1998, ApJ, 493, 613

Vaughan, S., Iwasawa, K., Fabian, A. C., \& Hayashida, K. 2005, MNRAS, 356, 524

Veilleux, S., \& Osterbrock, D. E. 1987, ApJS, 63, 295

Volonteri, M. 2010, A\&A Rev., 18, 279

Volonteri, M., Lodato, G., \& Natarajan, P. 2008, MNRAS, 383, 1079

Volonteri, M., \& Natarajan, P. 2009, MNRAS, 400, 1911

Wetzel, A. R., Tinker, J. L., \& Conroy, C. 2012, MNRAS, 424, 232

Whittle, M. 1985, MNRAS, 213, 1

Wrobel, J. M., \& Ho, L. C. 2006, ApJ, 646, L95

Xue, Y. Q., Wang, S. X., Brandt, W. N., et al. 2012, ApJ, 758, 129

Yan, R. 2011, AJ, 142, 153

Yan, R., \& Blanton, M. R. 2012, ApJ, 747, 61

Yee, H. K. C. 1980, ApJ, 241, 894

York, D. G., et al. 2000, AJ, 120, 1579 